\title{
Role of Apolipoprotein L1 in Human Parietal Epithelial Cell Transition
}

Vinod Kumar, ${ }^{*}$ Himanshu Vashistha, ${ }^{\dagger}$ Xiqian Lan, ${ }^{*}$ Nirupama Chandel, ${ }^{*}$ Kamesh Ayasolla, ${ }^{*}$ Seyedeh Shadafarin Marashi Shoshtari, ${ }^{*}$ Rukhsana Aslam, ${ }^{*}$ Nitpriya Paliwal, ${ }^{*}$ Frank Abbruscato, ${ }^{\dagger}$ Joanna Mikulak, Waldemar Popik, Mohamed G. Atta," Praveen N. Chander," Ashwani Malhotra, ${ }^{*}$ Catherine Meyer-Schwesinger, ${ }^{* *}$ Karl Skorecki, ${ }^{\dagger \dagger}$ and Pravin C. Singhal*

From the Immunology and Inflammation Center,* Feinstein Institute for Medical Research and Zucker School of Medicine at Hofstra-Northwell, Manhasset, New York; the Institute of Translational Research, ${ }^{\dagger}$ the Ochsner Clinic, New Orleans, Louisiana; the Humanitas Clinical and Research Center, ${ }^{\ddagger}$ Rozzano, Milan, Italy; Health Disparities and HIV ${ }^{\S}$ Meharry Medical College, Nashville, Tennessee; the Nephrogy Division, " Johns Hopkins Hospital, Baltimore, Maryland; the Department of Pathology, ${ }^{N}$ New York Medical College, Valhalla, New York; Nephrology Division, ** University of Hamburg, Hamburg, Germany; and Technion-Israel Institute of Technology, ${ }^{\dagger \dagger}$ Rambam Health Care Campus, Haifa, Israel

Accepted for publication July 2, 2018.

Address correspondence to Pravin C. Singhal, M.D., Immunology and Inflammation Center, Feinstein Institute for Medical Research and Zucker School of Medicine at HofstraNorthwell, 350 Community Dr., Manhasset, NY 11030. E-mail: psinghal@northwell.edu.

\begin{abstract}
Human parietal epithelial cells (PECS) are progenitor cells that sustain podocyte homeostasis. We hypothesized that the lack of apolipoprotein (APO) L1 ensures the PEC phenotype, but its induction initiates PEC transition (expression of podocyte markers). APOL1 expression and down-regulation of miR193a coincided with the expression of podocyte markers during the transition. The induction of APOL1 also stimulated transition markers in human embryonic kidney cells (cells with undetectable APOL1 protein expression). APOL1 silencing in PECs up-regulated miR193a expression, suggesting the possibility of a reciprocal feedback relationship between APOL1 and miR193a. HIV, interferon- $\gamma$, and vitamin D receptor agonist down-regulated miR193a expression and induced APOL1 expression along with transition markers in PECs. Luciferase assay suggested a putative interaction between miR193a and APOL1. Since silencing of APOL1 attenuated HIV-, vitamin D receptor agonist-, miR193a inhibitor-, and interferon- $\gamma-$ induced expression of transition markers, APOL1 appears to be a critical functional constituent of the miR193aAPOL1 axis in PECs. This notion was confirmed by further enhanced expression of PEC markers in APOL1 mRNA-silenced PECs. In vivo studies, glomeruli in patients with HIV, and HIV/APOL1 transgenic mice had foci of PECs expressing synaptopodin, a transition marker. APOL1 likely regulates PEC molecular phenotype through modulation of miR193a expression, and APOL1 and miR193a share a reciprocal feedback relationship. (Am J Pathol 2018, 188: 2508-2528; https://doi.org/10.1016/j.ajpath.2018.07.025)
\end{abstract}

Apolipoprotein L1 (APOL1) is a minor component of circulating lipid-rich multiprotein complexes in certain primate species, including humans. ${ }^{1}$ The trypanolytic function of circulating ancestral wild-type APOL1 (G0) has been well recognized for many years, antedating discovery of the derived renal risk variants (described as G1 and G2) in certain population of Sub-Saharan African descent. ${ }^{1}$ It is expressed in liver, pancreas, kidney, brain, macrophages, and endothelial cells. ${ }^{1}$ In kidneys, APOL1 protein is expressed in podocytes and tubular and vascular smooth muscle cells. ${ }^{2,3}$ Recently, APOL1's differentiating property has been observed in podocytes and monocytes. ${ }^{4,5}$ It has been shown to preserve differentiation in podocytes in adverse milieus ${ }^{4}$ and participates in monocytes' differentiation to M1 macrophages. ${ }^{5}$ On the other hand, APOL1 variants (G1 and G2) have been associated with a higher rates of the development of chronic diseases. ${ }^{6-12}$

PEC transition to podocytes has been demonstrated in juvenile mice. ${ }^{13,14}$ Nonetheless, the role of PEC transition in adult mice is controversial. ${ }^{15-19}$ Because mice do not express APOL1, the role of APOL1 was not evaluated in these

Supported by NIH grants RO1DK098074 and RO1DK118017 (P.C.S.) the Israel Science Foundation and Rambam Medical Center, Kaylie Kidney Health Center of Excellence, and the Beutler Foundation For Genomic Medicine Research (K.S.).

Disclosures: None declared. 
studies. The transition of human PECs to podocytes has been demonstrated in in vitro studies. ${ }^{20}$

The knockdown of miR193a stimulated the expression of podocyte molecular markers in PECs. ${ }^{20}$ Because APOL1 has a potential to act as a differentiating factor in human podocytes ${ }^{4}$ we hypothesize that modulation of the APOL1miR193 axis would induce PEC transition to podocytes.

Parietal epithelial cells line the inside of Bowman's capsule and continue with the proximal tubular epithelial cells at the urinary pole and with the podocytes at the vascular pole. ${ }^{21}$ PECs, proximal tubular cells, and podocytes originate from a common mesenchymal lineage and undergo divergent differentiation during embryogenesis. Podocytes and proximal tubular epithelial cells are highly differentiated cells and participate in the maintenance of the filtration barrier and water/solute transport, respectively. However, no specific function other than sustaining the integrity of Bowman's capsule was known for PECs, until recently. ${ }^{21}$ PECs are now considered progenitor cells for replacement of lost podocytes. ${ }^{13} \mathrm{We}$ previously reported that knockdown of miR193a in PECs initiated their transition in in vitro studies. ${ }^{20} \mathrm{We}$ also demonstrated that APOL1 inversely regulates miR193a expression in human podocytes and that expression of APOL1 is critical in differentiated human podocytes to protect dedifferentiation in adverse milieus. ${ }^{4}$ We now hypothesize that APOL1 and miR193a form a reciprocally linked feedback loop to regulate PEC phenotype in humans. In this loop, lack of APOL1 is necessary for the optimized PEC phenotype. However, its presence in human PECs is dispensable as a trade-off for the compromised podocyte renewal.

In the present study, we examined the role of the APOL1miR193a axis in dynamics of PEC molecular phenotype kinetics. We also investigated the effects of the presence or the absence of APOL1 protein in PECs and in cells with undetectable APOL1 protein expression on their molecular phenotype as well as induction of transition markers. We have used renal biopsy specimens of patients with HIV-associated nephropathy (HIVAN) to display that HIV infection has the potential to induce APOL1 expression in vivo.

\section{Materials and Methods}

\section{Human PECs, HEK Cells, and HepG2 Cells}

Immortalized human PECs were obtained from Prof. Catherine Meyer-Schwesinger (University Medical Center, Hamburg-Eppendorf, Hamburg, Germany). Details of the

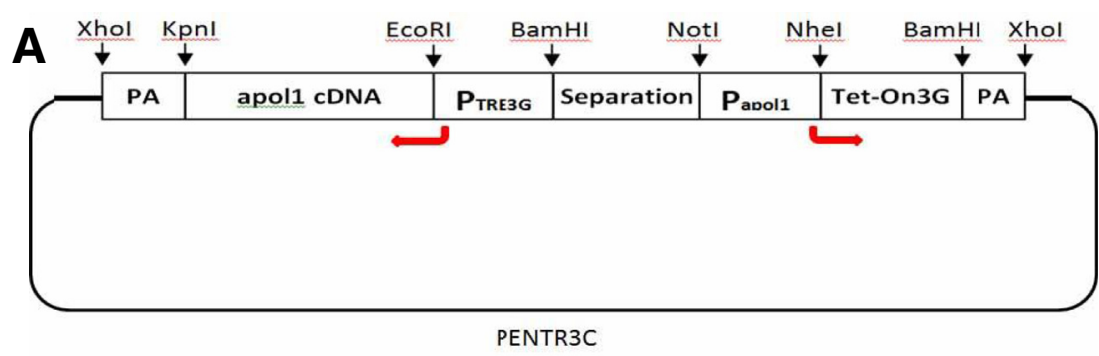

B

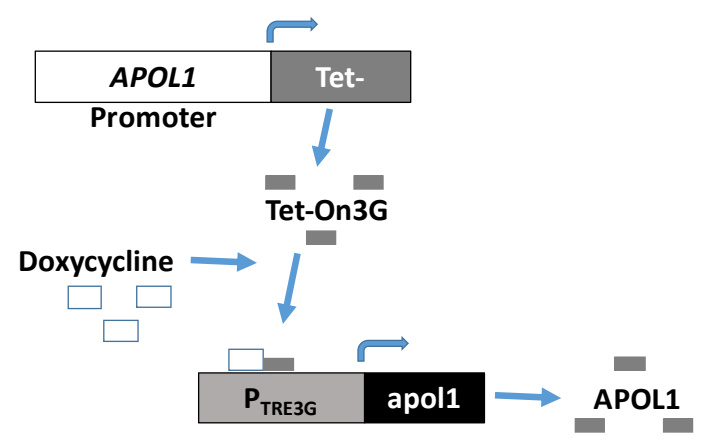

C
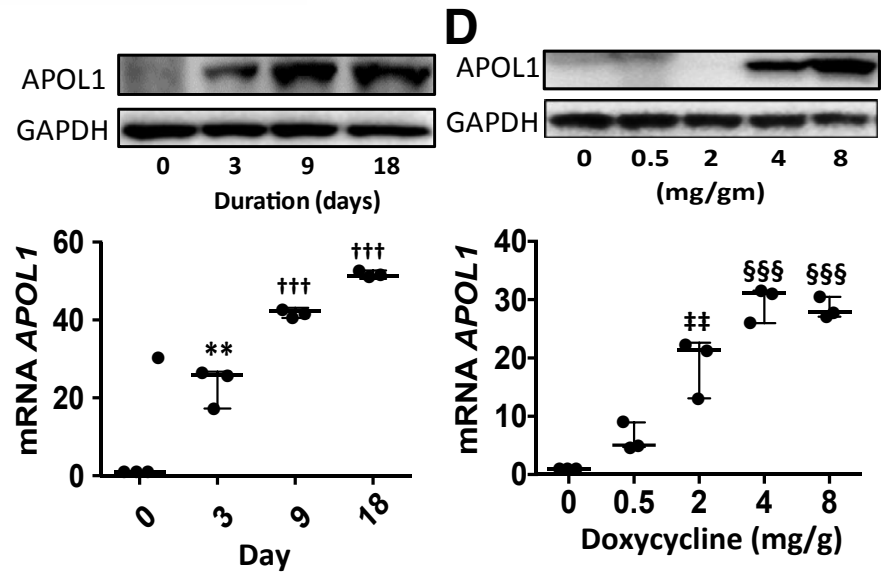

Figure 1 Generation of apolipoprotein (APO) L1 transgenic mice. A: A doxycycline-inducible APOL1 (G1) construct was made by using plasmid pENTR3C as the backbone. B: The cDNA of Tet0n3G and its promoter PTRE3G were used from Tet-0n 3G Tetracycline-Inducible Expression Systems. The working flowchart is shown. C: To examine the time course effect of APOL1 protein expression, 4-week-old APOL1 (G1) transgenic mice were fed doxycycline (2.5 mg/kg body weight daily for 3 consecutive days and then every other day) for different periods ( $0,3,9$, and 18 days). Kidneys were harvested. Proteins and RNAs were extracted. Protein blots of renal tissues were probed for APOL1 and reprobed for glyceraldehyde-3-phosphate dehydrogenase (GAPDH). Representative gels are displayed. cDNAs were amplified for APOL1 mRNA. Renal tissues displayed the expression of APOL1 protein and mRNA in a time course manner. D: To assess the dose-response effect, 4-week-old APOL1 (G1) transgenic mice were administered different doses of doxycycline for 3 consecutive days. Kidneys were harvested, and proteins and RNAs were extracted. Protein blots of renal tissues were probed for APOL1 and reprobed for GAPDH. Representative gels are displayed. cDNAs were amplified for APOL1 mRNA. Doxycycline enhances the expression of the APOL1 protein and mRNA in a dose-dependent manner. $n=3 .{ }^{* * P}<0.01$ versus 0 and 18 days; ${ }^{\dagger \dagger} P<0.001$ versus 0 day; ${ }^{\ddagger \ddagger} P<0.01$ versus 0 and $0.5 \mathrm{mg}$ and ${ }^{\S \S \S} \mathrm{P}<0.001$ versus $0,0.5$, and 2 mg. $P A$, polyadenylation. 
characteristics of PECs have been reported previously. ${ }^{20}$ PECs were grown in 1:1 endothelial growth medium supplemented with $5 \%$ fetal calf serum, $0.4 \%$ bovine brain extract, $0.1 \%$ human epidermal growth factor, $0.1 \%$ hydrocortisone, $0.1 \%$ gentamicin and amphotericin $\mathrm{B}, 100 \mathrm{U} /$ $\mathrm{mL}$ of penicillin, $100 \mathrm{mg} / \mathrm{mL}$ of streptomycin (Lonza, Basel, Switzerland), and RPMI 1640 medium supplemented with $10 \%$ fetal calf serum and $1 \times$ ITS (insulin, transferrin, and selenium) at $33^{\circ} \mathrm{C}$ (5\% carbon dioxide incubator). For transition (differentiation), PECs were cultured for 14 days in endothelial growth medium and RPMI 1640 medium with the added supplement described above. During the transition, PECs were seeded on laminin- or fibronectincoated dishes (BD Biocoat Cellware; BD Biosciences, Franklin Lakes, NJ), and the medium was supplemented with a special induction medium that contained vitamin $\mathrm{D}_{3}$ (100 nmol/L; Sigma-Aldrich, St Louis, MO), retinoic acid (100 $\mu \mathrm{mol} / \mathrm{L} ;$ Sigma-Aldrich), and dexamethasone (0.1

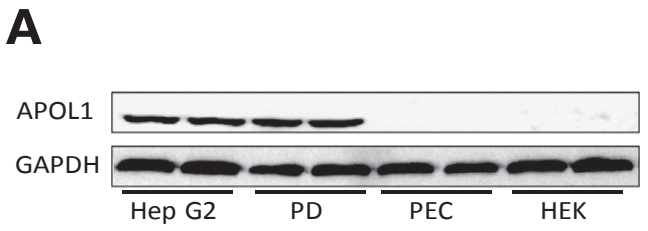

B
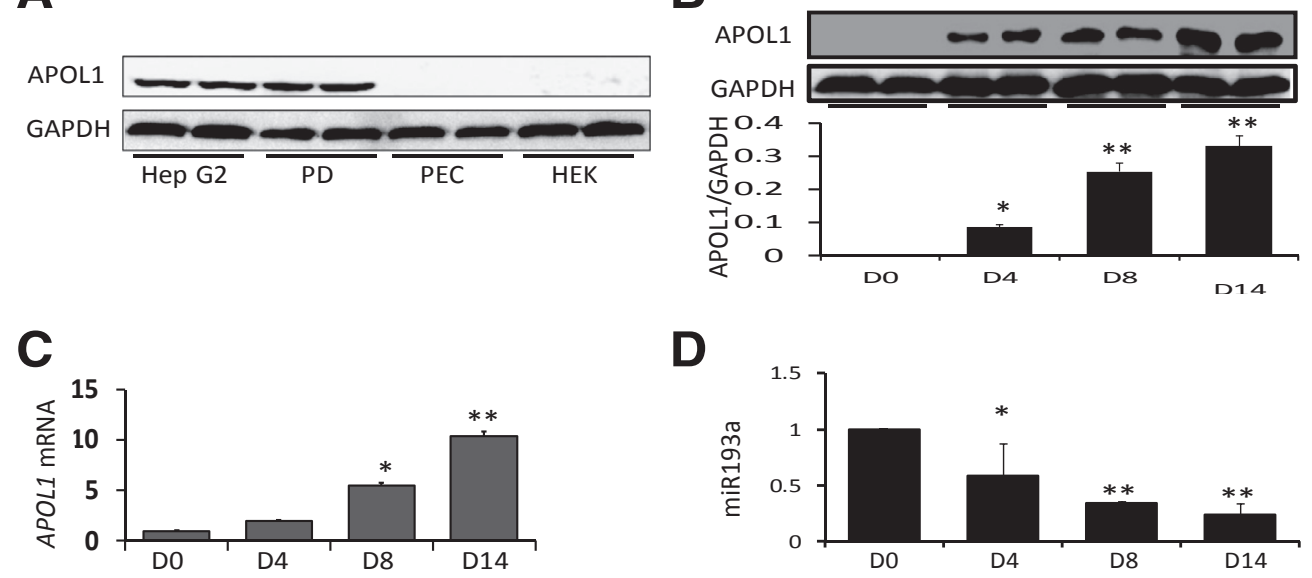

D
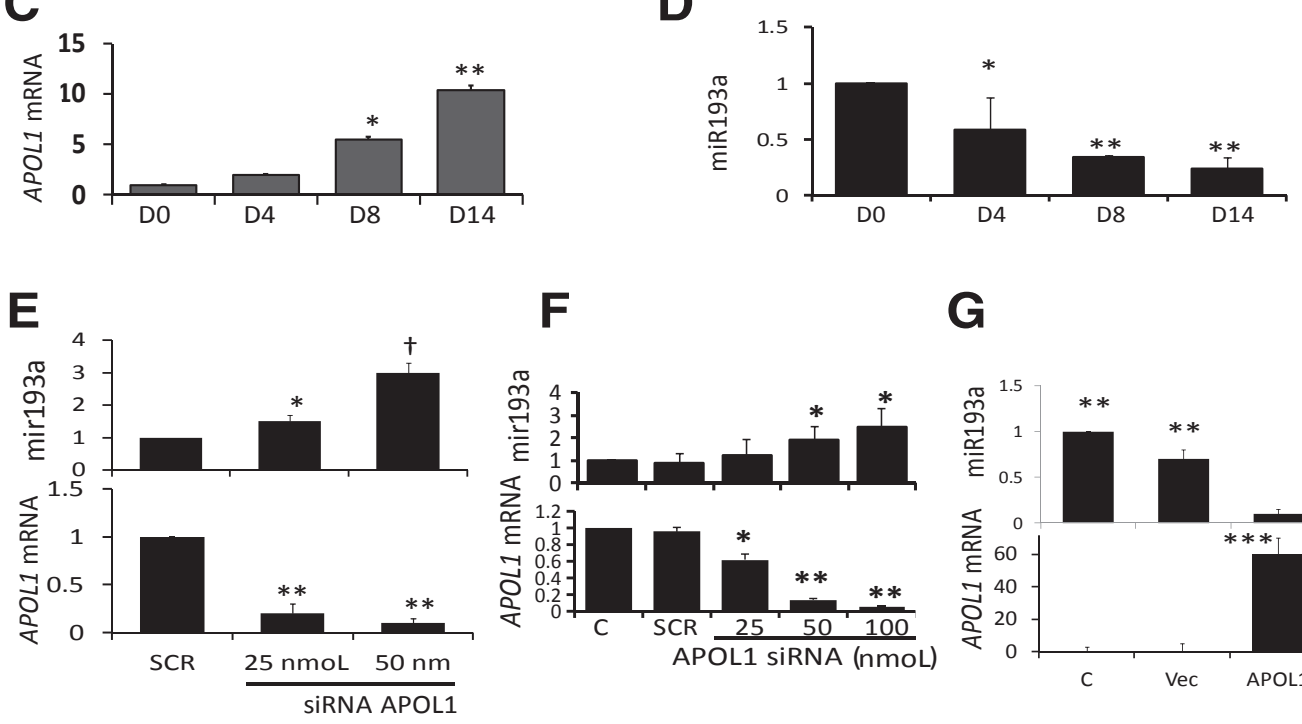

G

H

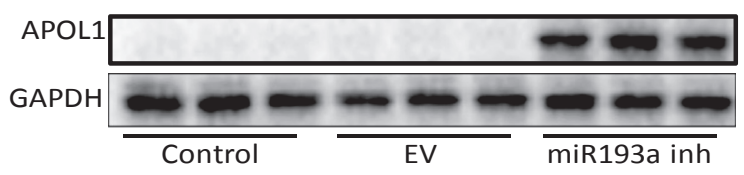

J

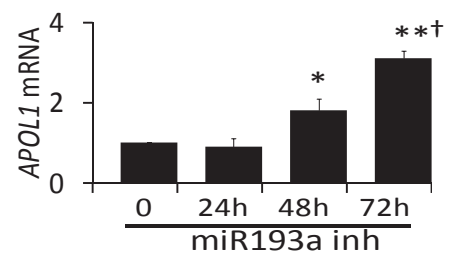

I

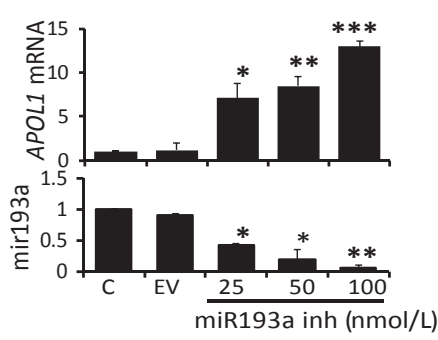

$\mathbf{K}$

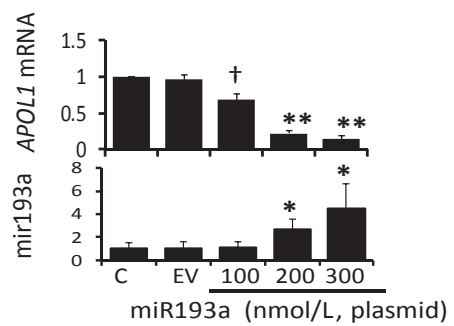


$\mu \mathrm{mol} / \mathrm{L}$; Sigma-Aldrich). DNA sequencing of PECs revealed APOL1G0 phenotype.

Human embryonic kidney (HEK) cells were purchased from ATCC (Manassas, VA) (catalog number CRL1573TM) and cultured in Dulbecco's modified Eagle's medium supplemented with $10 \%$ fetal calf serum and $25 \mathrm{mmol} / \mathrm{L}$ HEPES. HepG2 cells (hepatocyte cell line) were gifted by Prof. Sanjeev Gupta (Liver Research Center, Albert Einstein College Medicine, New York, NY). These cells were cultured in Dulbecco's modified Eagle's medium supplemented with $10 \%$ fetal calf serum and $25 \mathrm{mmol} / \mathrm{L}$ HEPES.

\section{Production of Pseudotyped Retroviral Supernatant}

The GFP reporter gene (from pEGFP-C1; Clontech, Palo Alto, CA) was substituted instead of gag/pol genes in HIV-1 proviral construct pNL4-3 as described in our previous publications. $^{22-24}$ The same construct has been used to generate HIV transgenic mice (Tg26). ${ }^{25,26}$ This parental construct (pNL4-3: $\Delta$ G/P-GFP) was used to produce vesicular stomatitis virus $\mathrm{G}$-pseudotyped viruses to provide pleiotropism and high-titer virus stocks. Infectious viral supernatants were generated from transient transfection of 293 T cells using Effectene (Qiagen Inc., Hilden, Germany). The HIV-1 gag/pol and VSV.G envelope genes were provided in trans using pCMV R8.91 and pMD.G plasmids, respectively. As a negative control, the virus was also produced from pHR-CMV-IRES2-GFP- $\Delta \mathrm{B}$, which contained HIV-1 long-term repeats and green fluorescent protein (GFP) empty expression vector. The viral stocks were titrated by infecting $293 \mathrm{~T}$ cells with 10 -fold serial dilution. The reciprocal of the lowest dilution showing expression of GFP was defined as GFP-expressing units (GEU) per milliliter. Viral stocks ranging from $10^{5}$ to $10^{6} \mathrm{GEU} \mathrm{m} / \mathrm{L}$ were obtained. Kidney cells transduced with HIV construct showed expression of all HIV genes except gag/pol. ${ }^{22,25}$

\section{Generation and Identification of APOL1 Transgenic Mice}

The TetOn3G-APOL1G0 construct was released from the plasmid vector backbone by digestion with XhoI and purified by gel electrophoresis, and DNA was extracted using a QIAEX II Gel Extraction Kit (Qiagen). The purified construct DNA was introduced into the pronuclear of fertilized oocytes (from the FVB/N mouse) by microinjection using standard techniques. All experimental protocols were approved by the local committee for laboratory animal welfare of the Cold Spring Harbor Laboratory.

\footnotetext{
Figure 2 Apolipoprotein (APO) L1-miR193a axis in human parietal epithelial cells (PECs). A: Expression of APOL1 protein by the kidney and nonkidney cells. Protein blots of PECs, human embryonic kidney (HEK) cells, differentiated podocytes (PDs), and HepG2 cells were probed for APOL1 and reprobed for glyceraldehyde-3-phosphate dehydrogenase (GAPDH). Gels from two different lysates are displayed. PECs were evaluated for APOL1 phenotype; both PECs and HEK cells lack APOL1 protein expression. B: To differentiate PECs, undifferentiated PECs were incubated in special media for a different period (0, 4, 8, and 14 days) at $37^{\circ} \mathrm{C}$. Subsequently, protein blots were probed for the APOL1 and reprobed for GAPDH. Blots from two different lysates are displayed in the top panel. The bottom panel shows cumulative densitometric data. APOL1 expression emerged on day 4 and progressed with time. C: To determine the time course of APOL1 transcription during the transition, RNAs were extracted from cell lysates of the above-mentioned experiment. cDNAs were amplified with a specific primer to APOL1. APOL1 transcription went up during the transition. D: To evaluate the time course of miR193a levels during the transition, miR193a levels were assayed from RNAs extracted in C. Cumulative data are shown in the bar diagram. miR193a levels went down during PEC transition. E: Undifferentiated PECs were transfected with scrambled (SCR) or APOL1-siRNA (25 or $50 \mathrm{nmol} / \mathrm{L}$ ). RNAs were extracted. cDNAs were amplified with a primer specific for APOL1; cumulative data are shown in a bar diagram. The same RNAs were assayed for miR193a. APOL1 silencing in PECs up-regulates miR193a expression in a dosedependent manner. F: HepG2 cells were transfected with SCR, at different concentrations (25, 50, and100 nmol/L), or APOL1 siRNA. RNAs were extracted from control (C) and experimental cells. CDNAs were amplified with a primer specific for APOL1. Cumulative data are shown in the bar diagram. RNAs were assayed for miR193a. In HepG2 cells, the lack of APOL1 enhances miR193a levels in a dose-dependent manner. G: To evaluate the effect of AP0L1 induction on miR193a expression, vector (Vec) (lentivirus) and APOL1 lentivirus were transduced in PECs. RNAs were extracted from C, Vec, and APOL1-tranduced PECs. CDNAs were amplified with a primer specific for APOL1. miR193a levels were assayed from the extracted RNAs. Cumulative data of APOL1 mRNA are shown in the bottom panel and of miR193a levels in the top panel. APOL1 induction in PECs down-regulated miR193a expression. H: To determine the effect of miR193a down-regulation on the induction of APOL1, undifferentiated PECs (confluent at $33^{\circ} \mathrm{C}$ ) were transfected with empty vector (EV) or miR193a inhibitor (inh) and incubated at $37^{\circ} \mathrm{C}$ for 48 hours. Proteins were extracted from C-, EV-, and miR193 inh-transfected PECs. Protein blots were probed for AP0L1 and reprobed for GAPDH. Blots of three different lysates of control and experimental PECs are shown. Inhibition of miR193a induced the expression of APOL1 in PECs. I: To evaluate the dose-response effect of miR193a inhibition on APOL1 transcription, PECs (undifferentiated, 0 days) were transfected with EV or different concentrations of miR193a inhibitor (plasmid, 25, 50, and $100 \mathrm{nmol} / \mathrm{L}$ ). After 48 hours of incubation, RNAs were extracted from C and experimental cells. RNAs were assayed for miR193a. CDNAs were assayed with a primer specific for APOL1. miR193a levels are shown in the bottom panel, and APOL1 mRNA levels are shown in the top panel. Inhibition of miR193a enhances the transcription of APOL1 in a dose-dependent manner. J: To examine the time course effect of miR193a inhibition, PECs (undifferentiated, confluent) were incubated in media that contained a specific inhibitor of miR193a (25 nmol/L, plasmid) for different periods $(0,24,48$, and 72 hours). RNAs were extracted, and CDNAs were amplified for APOL1. Inhibition of miR193a enhances the transcription of APOL1 mRNA in a time course manner. K: To assess the presence of APOL1-miR193a axis in nonkidney cells expressing APOL1, HepG2 cells were transfected with EV or different concentrations of miR193a plasmid $(100,200$, and $300 \mathrm{nmol} / \mathrm{L})$. RNAs were extracted and assayed for miR193a from C and experimental cells. cDNAs were amplified with a primer specific for APOL1. mRNA193a levels are shown in the bottom panel, and APOL1 mRNA levels are displayed in the top panel. Up-regulation of miR193a down-regulates the transcription of $A P O L 1$ in a dose-dependent manner. $n=3(\mathbf{A}, \mathbf{E}-\mathbf{K}) ; n=4(\mathbf{B}$ and $\mathbf{C})$. ${ }^{*} P<0.05$ vs $D 0$ (B and D); ${ }^{* *} P<0.01$ versus D0 and D4 (B); ${ }^{*} P<0.05$ versus D0 and D4 (C); ${ }^{* * P}<0.01$ versus D0, D4, and D8 (C); ${ }^{* *} P<0.01$ versus D0 (D); ${ }^{*} P<0.05$ versus respective $\mathrm{SCR}(\mathbf{E}) ;{ }^{* *} P<0.01$ versus respective $\mathrm{SCR}(\mathbf{E}) ;{ }^{\dagger} P<0.01$ versus respective $\mathrm{SCR}$ and $25 \mathrm{nmol} / \mathrm{L}$ siRNA AP0L1 (E); ${ }^{*} P<0.05$ versus respective $\mathrm{C}$ and SCR (F); ${ }^{* *} P<0.01$ versus respective $C, S C R$, and $25 \mathrm{nmol} / \mathrm{L}$ siRNA APOL1 (F); ${ }^{*} P<0.01$ versus AP0L1 (G); ${ }^{* *} P<0.001$ versus $C$ and Vec $(\mathbf{G})$; ${ }^{*} P<0.05$ versus respective $\mathrm{C}$ and $\mathrm{EV}(\mathbf{I}$ and $\mathbf{K}) ;{ }^{\dagger} P<0.05$ versus respective $\mathrm{C}$ and $\mathrm{EV}(\mathbf{K}) ;{ }^{* *} P<0.01$ versus respective $\mathrm{C}$ and $\mathrm{EV}(\mathbf{I}) ;{ }^{* * *} P<0.001$ versus respective $\mathrm{C}$ and $\mathrm{EV}(\mathbf{I}) ;{ }^{*} P<0.05$ versus 0 and 24 hours $(\mathbf{J}) ;{ }^{* *} P<0.01$ versus 0 and 24 hours $(\mathbf{J}) ;{ }^{\dagger} P<0.05$ versus 48 hours $(\mathbf{J})$; ${ }^{*} P<0.05$ versus respective C, EV, and $100 \mathrm{nmol} / \mathrm{L}$ miR193a (K); ${ }^{*} P<0.01$ versus respective C, EV, and $100 \mathrm{nmol} / \mathrm{L} \mathrm{miR193a(K).} \mathrm{D,} \mathrm{day.}$
} 
Transgenic mice were identified by PCR on tail genomic DNA (50 to $100 \mathrm{ng}$ ), using Tg- FW (5'-ATCTCAGCTGAAAGCGGTGAAC- $3^{\prime}$ ) and Tg-RV (5'-GGTACCTCACAGTTCTTGGTCCGCCTGCAGAATC- $\left.3^{\prime}\right)$ primers and 30 cycles of $95^{\circ} \mathrm{C}$ (30 seconds), $57^{\circ} \mathrm{C}$ (30 seconds), and $72^{\circ} \mathrm{C}$ (30 seconds), and a final extension of $72^{\circ} \mathrm{C}$ for 5 minutes. The reaction was performed with Taq DNase polymerase (Qiagen) in a total reaction volume of $20 \mu \mathrm{L}$, including $400 \mathrm{nmol} / \mathrm{L}$ of both primers. Samples were analyzed by standard agarose gel electrophoresis $(1.5 \%$ gel $)$
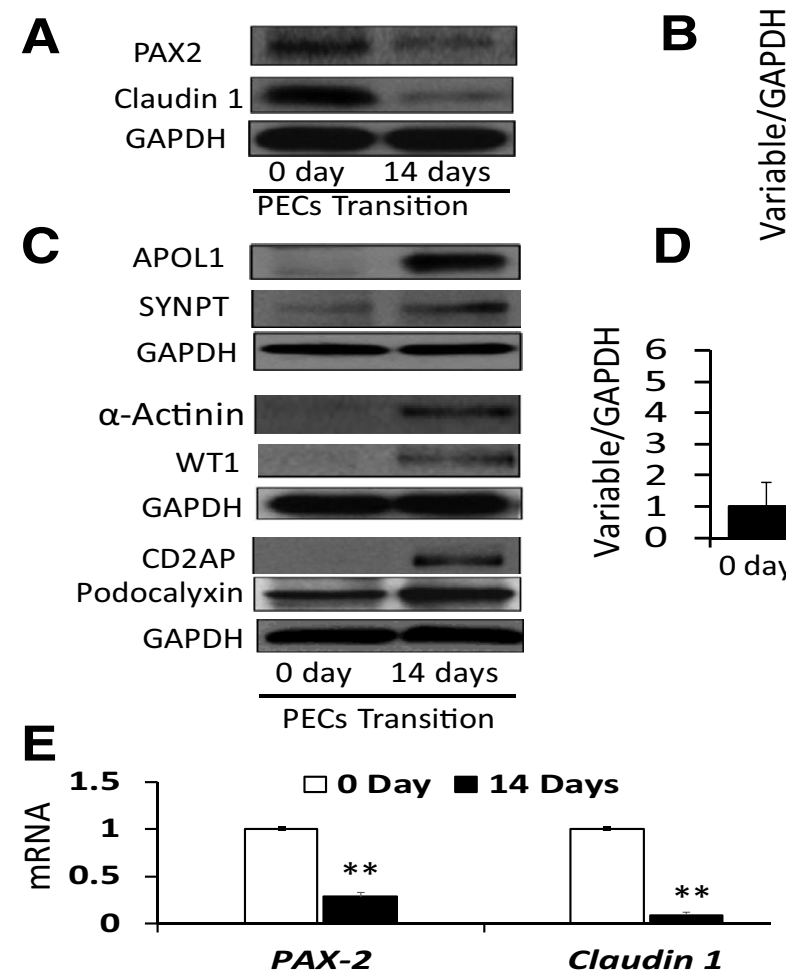

G

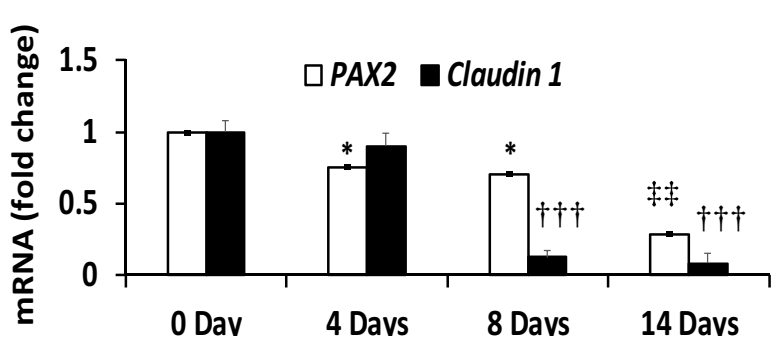

I
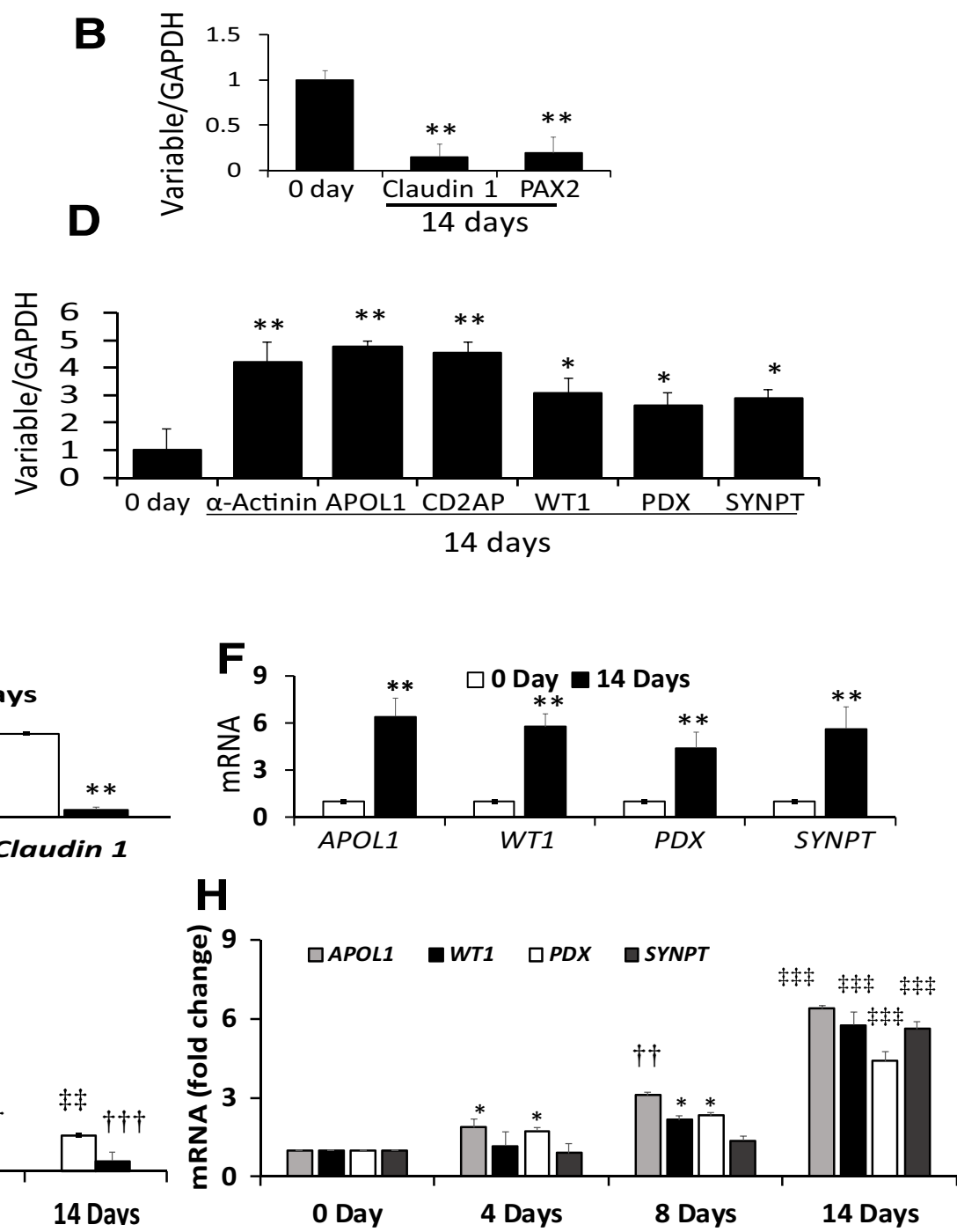

a-Actinin $\quad$ PDX

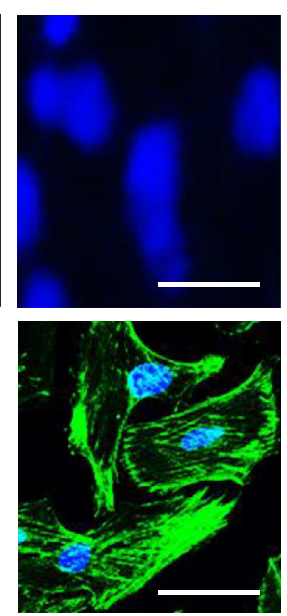

WT1
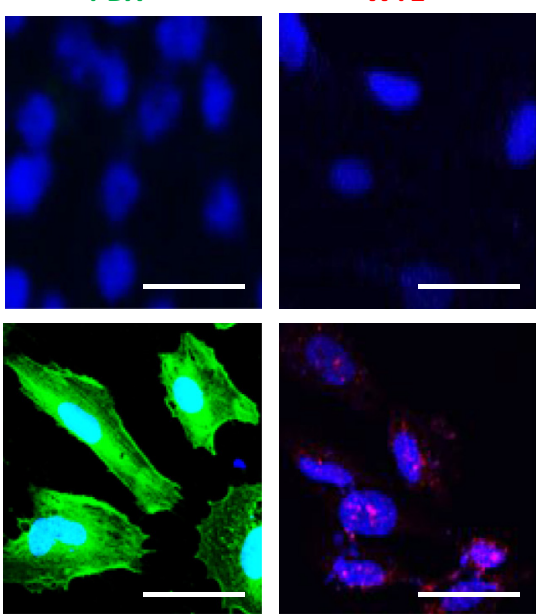
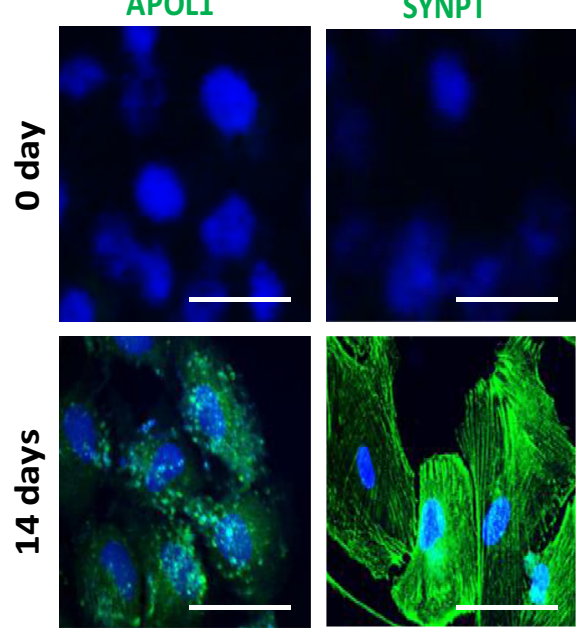


\section{HIV (Tg26):AP0L1 Transgenic Mice}

APOL1 transgenic mice were cross-bred with Tg26 (HIV transgenic) mice, a gift from Prof. Paul Klotman (University of Texas, Houston). In the generation of these mice, HIV construct (pNL4-3) was used. ${ }^{25,26} \mathrm{Tg} 26$ mice and APOL1 transgenic mice are on FVB/N background. HIV transgene is constitutively expressed, but APOL1 expression was induced by doxycycline containing feed $(200 \mathrm{mg} / \mathrm{kg}$, BioServe, Beltsville, MD). Animal studies were approved by the Center for Comparative Physiology of Feinstein Institute for Medical Research, Northwell Health, New York.

\section{Human Renal Biopsy Data}

Archived biopsy specimens from the patients of HIVAN (Long Island Jewish Medical Center, New Hyde Park, NY; Johns Hopkins Hospital, Baltimore, MD; Westchester Medical Center, Valhalla, NY) and control patients (renal transplant kidney donors) were used. The institutional review board of Feinstein Institute for Medical Research, Northwell Health, New York, approved the use of these archived biopsy tissues.

\section{Immunofluorescence of PECs and HEK Cells}

Cells were grown on fibronectin-coated coverslips. After differentiation or inductions, cells were washed with $1 \times$ phosphate-buffered saline (PBS) and fixed in $4 \%$ paraformaldehyde for 10 minutes at room temperature. Cells were washed three times with $1 \times$ PBS and permeabilized with $0.5 \%$ Triton X-100. After another washing step with $1 \times$ PBS, cells were incubated in $5 \%$ bovine serum albumin for 30 minutes at room temperature. Cells were incubated with the following primary antibodies: APOL1 (1G12D11; $1.57 \mu \mathrm{g} / 100 \mu \mathrm{L}$; Proteintech, Chicago, IL), rabbit (rb)PAX2 (EP3251; $0.145 \mu \mathrm{g} / 100 \mu \mathrm{L}$, Abcam, Cambridge, UK), rb-claudin 1 (0.175 $\mu \mathrm{g} / 100 \mu \mathrm{L}$, Abcam), rb-Wilms tumor 1 (WT1) $(0.36 \mu \mathrm{g} / 100 \mu \mathrm{L}$, Abcam), rb-podocalyxin $(0.53 \mu \mathrm{g} / 100 \mu \mathrm{L}$, catalog number PAI-46170; Life Technologies, Carlsbad, CA), synaptopodin (P-19, $0.36 \mu \mathrm{g} / 100$ $\mu L$, Santa Cruz Biotechnology Inc., Santa Cruz, TX), CD2AP (B-4, $0.36 \mu \mathrm{g} / 100 \mu \mathrm{L}$, Santa Cruz Biotechnology), and mouse- $\alpha$-actinin $(\mathrm{H}-2 ; 0.1 \mu \mathrm{g} / 100 \mu \mathrm{L}$, Santa Cruz Biotechnology) for overnight at $4^{\circ} \mathrm{C}$. After incubation, cells were washed three times with $1 \times$ PBS and stained with fluorochrome [AF488 (green) and AF594 (red), $1 \mu \mathrm{g} / 200$ $\mu \mathrm{L}]$ conjugated secondary antibody to goat anti-rabbit and donkey anti-mouse $(0.1 \mu \mathrm{g} / 200 \mu \mathrm{L}$, Molecular Probes, Eugene, OR) for 30 minutes at room temperature and nuclear stain Hoechst 33342. For each set of experiment, the negative control was prepared with the use of isotype $\operatorname{IgG}$ in place of primary antibody followed by secondary antibody. Staining was visualized under a confocal microscope.

\section{Immunofluorescence of Kidney Tissue}

Human renal biopsy slides (from control patients and patients with HIVAN) and slides of renal cortical sections from FVB/N (control) and HIV:APOL1 transgenic (Tg26: APOL1) mice were immersed into $100 \%$ xylene for 5 minutes $(4 \times)$; then slides were dipped in $100 \%$ ethanol for 5 minutes twice, then into $70 \%$ ethanol 5 minutes twice, and finally in $50 \%$ ethanol for 5 minutes twice. Slides were washed with double distilled water for 1 minute. Afterward, slides were subjected to a $1 \times$ retrieve-all antigen unmasking system buffer (Covance, Dedham, MA) at $100^{\circ} \mathrm{C}$ for 90 minutes. Slides were exposed at room temperature for 20 to 30 minutes for cooling. After the slides were washed with $1 \times$ PBS, they were subjected to $0.3 \%$ Triton X-100 for 20 minutes at room temperature and blocked with $2 \%$ bovine serum albumin for 2 hours. After blocking, primary antibodies [pan-cytokeratin, guinea pig (serum), catalog number BP5069, Acris at 1:50 dilution; synaptopodin, rabbit polyclonal, catalog number SC5049 (H-140) Santa Cruz Biotechnology, $4 \mu \mathrm{g} / \mathrm{mL}$; and APOL1, mouse monoclonal,

\footnotetext{
Figure 3 The apolipoprotein (APO) L1 expression is associated with the expression of parietal epithelial cells (PECs) transition markers. A: To evaluate PEC markers in transited PECs, undifferentiated PECs were incubated in special media for 14 days at $37^{\circ} \mathrm{C}$. Protein blots of control (undifferentiated, 0 days) and differentiated (transited) PECS (14 days) were probed for PAX2 and reprobed for claudin 1 and glyceraldehyde-3-phosphate dehydrogenase (GAPDH). Representative gels are displayed. B: Cumulative densitometric data from protein blots from $\mathbf{A}$ are displayed in a bar diagram. PEC transition is associated with down-regulation of PAX2 and claudin 1 expression. C: To assess transition markers, protein blots from cellular lysates of undifferentiated (0 days) and differentiated (14 days) PECs (from A) were probed for APOL1 and reprobed for synaptopodin (SYNPT) and GAPDH. Protein blots from the same lysates were probed for $\alpha$-actinin and reprobed for Wilms' tumor 1 (WT1) and GAPDH. Protein blots were also probed for CD2AP and reprobed for podocalyxin (PDX) and GAPDH. Representative gels are displayed. D: Cumulative densitometric data of protein blots generated in C are shown in a bar diagram. PEC transition manifests in the form of enhanced expression of podocyte markers. E: To evaluate transcription of PEC markers in transited PECs, RNAs were extracted from the lysates of undifferentiated ( 0 days) and differentiated (14 days) PECs (from 3A). cDNAs were amplified with specific primers for PAX2 and claudin 1 . Transited PECs displayed an attenuated transcription of PEC markers. F: To determine the transcription of transition markers in transited PECs, cDNAs from $\mathbf{E}$ were amplified with specific primers for APOL1, WT1, PDX, and SYNPT. Transited PECs display an enhanced transcription of transition markers. G: To determine the time course effect on the transcription of PEC markers during the transition, PECs were incubated in media for variable periods $\left(0,4,8\right.$, and 14 days) at $37^{\circ} \mathrm{C}$. RNAs were extracted, and CDNAs were amplified with specific primers for PAX2 and claudin 1. The transcription of PEC markers deceases during the transition in a time course manner. H: To evaluate the time course effect on the transcription of PECs transition markers, cDNAs obtained from the $2 \mathrm{G}$ (RNAs) were amplified with specific primers for APOL1, WT1, PDX, and SYNPT. The transcription of transition markers increases during the transition in a time course manner. I: PECs grown on coverslips were fixed on 0 (undifferentiated) and 14 days (differentiated) and labeled for APOL1, SYNPT, $\alpha$-actinin, PDX, and WT1. Subsequently, PECs were examined under a confocal microscope. Representative fluoromicrographs (APOL1, SYNPT, $\alpha$-actinin, and PDX displayed green and WT1 exhibited red fluorescence) are displayed. $n=4(\mathbf{A}$ and $\mathbf{C}-\mathbf{F})$. ${ }^{\star} P<0.05,{ }^{*} P<0.01$ versus respective 0 days; ${ }^{\dagger \dagger} P<0.01$ ersus respective 0 and 4 days; ${ }^{\ddagger \ddagger} P<0.01,{ }^{\ddagger \ddagger} P<0.001$ versus respective 0,4 , and 8 days. Scale bars $=50 \mu \mathrm{m}$.
} 
catalog number 66124-1-1G, Proteintech, $125 \mu \mathrm{g} / \mathrm{mL}]$ were added overnight at $4{ }^{\circ} \mathrm{C}$. The following day, slides were washed with $0.1 \%$ Triton $\mathrm{X}-100$ for 5 minutes thrice on a shaker at room temperature. The secondary antibody was added with fluorescence conjugated at 1:500 dilutions for 1 hour at room temperature. Nuclei were stained with DAPI. Slides were washed with $0.1 \%$ Triton X-100 for 5 minutes thrice on a shaker at room temperature and mounted for confocal microscopy.

Images of APOL1, cytokeratin, and synaptopodin staining were captured on the same $z$ axis using SlideBook software version 6.09 (Intelligent Imaging Innovations Inc., Denver, $\mathrm{CO})$. Image analysis and quantification were performed using Broad Institute's CellProfiler suite 3.0.0 (Broad Institute, Cambridge, MA). ${ }^{27}$ Briefly, images were converted from color to gray, and primary objects were identified as 10- to 60pixel objects in DAPI channel and 5- to 200-pixel objects for APOL1, cytokeratin, and synaptopodin channels. Identified objects of APOL1 were masked with the objects identified for cytokeratin to determine APOL1 objects labeled with cytokeratin. Gray channel of synaptopodin was combined separately with APOL1 and cytokeratin to generate combined channel images. Primary objects identified in the combined image of synaptopodin with APOL1 were masked by the primary objects identified in the combined image of synaptopodin with cytokeratin to determine synaptopodin objects labeled with APOL1 and cytokeratin. Binary outlines were generated, the area occupied by cytokeratin labeled with APOL1 or the area occupied by cytokeratin labeled with APOL1, and synaptopodin was measured. ${ }^{28}$ All measurements were exported directly to CSV files and were subsequently analyzed using GraphPad Prism software 7 (GraphPad Software, San Diego, CA) to generate dot plots.

\section{Real-Time Quantitative PCR}

Total RNA was isolated from human PECs, HEK cells, HepG2 cells, and human podocytes with TRIzol reagent (Invitrogen, Carlsbad, CA). cDNA was synthesized using $1 \mu \mathrm{g}$ of total RNA treated with a High-Capacity cDNA Reverse Transcription kit (Thermo Fisher Scientific, Waltham, MA) according to the manufacturer's instructions. Real-Time PCR was performed using one-step iTaqTM Universal Syber Green kit (Bio-Rad Laboratories, Hercules, CA) using specific
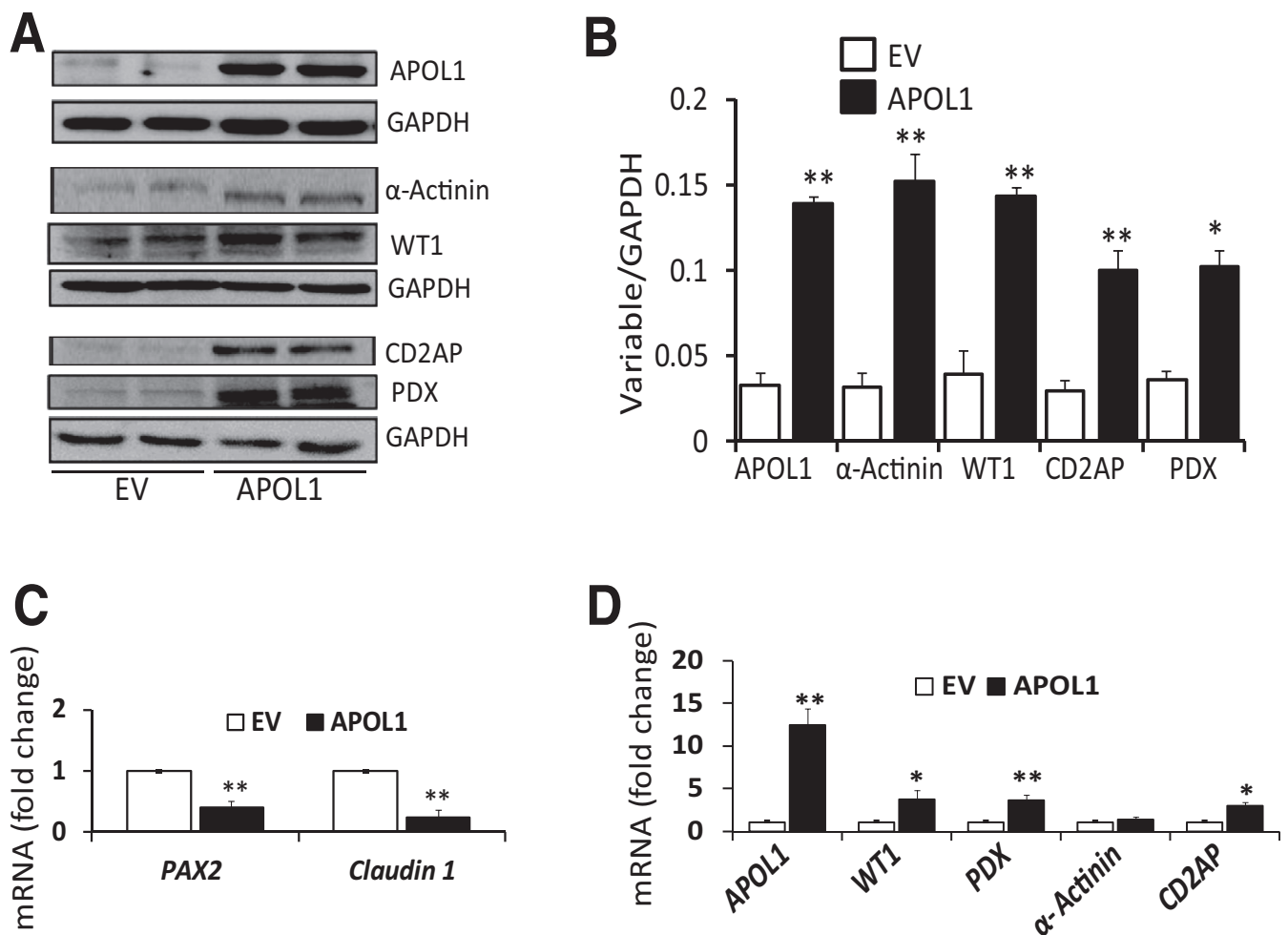

Figure 4 Induction of apolipoprotein (APOL) 1 expression in human embryonic kidney (HEK) cells induces the expression of transition markers. A: To determine APOL1 induction in kidney cells with undetectable APOL1 protein expression, HEK cells were transfected with empty vector (EV) or APOL1 plasmids. Protein blots were probed for APOL1 and reprobed for glyceraldehyde-3-phosphate dehydrogenase (GAPDH). The same cellular lysates were probed for $\alpha$ actinin, Wilms' tumor 1 (WT1), CD2AP, and podocalyxin (PDX) and reprobed for GAPDH. Gels from two different lysates are displayed. B: Cumulative densitometric data from the gels generated from the above-mentioned lysates are displayed. APOL1 induction in parietal epithelial cells (PECs) is associated with the expression of transition markers in HEK cells. C: To determine the effect of APOL1 induction on PEC markers in HEK cells, RNAs were extracted from the above-mentioned lysates. cDNAs were amplified for PAX2 and claudin 1. Cumulative data are shown. APOL1 induction in HEK cells down-regulates the transcription of PEC markers. D: To assess the effect of APOL1 induction on PEC transition markers in HEK cells, RNAs were extracted from the above-mentioned lysates. cDNAs were amplified for APOL1, WT1, PDX, $\alpha$-actinin, and CD2AP. Cumulative data are shown in a bar diagram. The induction of APOL1 in HEK cells is associated with enhanced transcription of transition markers in HEK cells. $n=4$ (A). ${ }^{*} P<0.05,{ }^{*} P<0.01$ versus respective EV. 
primers obtained from Life Technologies (Thermo Fisher Scientific): Glyceraldehyde-3-phosphate dehydrogenase (GAPDH) forward: 5'-CCCATCACCATCTTCCAGGAG-' 3 ; reverse: 5'-GTTGTCATGGATGACCTTGGC-' 3 ; WT1: forward: 5'-CGAGAGCGATAACCACACAACG-'3, reverse: 5'-GTCTCAGATGCCGACCGTACAA-'3; synaptopodin: forward: 5'-CTTCTCCGTGAGGCTAGTGC-' 3 , reverse: $5^{\prime}$-TGAGAAAGGCTTGAAAGG- ${ }^{\prime} 3$; podocalyxin: forward: $\quad 5^{\prime}$-TGTTTTGTTAGATGAGTCCGTAGTA- ${ }^{\prime} 3$, reverse: $5^{\prime}$-CGCTGCTACTGTCA- ${ }^{\prime} 3$; $\alpha$-actinin-4: forward: 5'-GTTCTCGATCTGTGTGCCTG-' 3 , reverse: $5^{\prime}$-GACC TGGACCC- 3 ; claudin 1: forward: $5^{\prime}$-TGGGGGTGCGATATTTCTTC- ${ }^{\prime} 3$, reverse: 5'-CCTCCCAGAAGGCAGAGAGA-'3; PAX2 forward: 5'-GGCTGTGTCAGCA-AAATCCTG-' 3 , reverse: 5'-TCCGGATGATTCTGTTGATGG-'3; APOL1 forward: 5'-ATCTCAGCTGAAAGCG-GTGAAC- ${ }^{\prime} 3$, reverse: 5'-TGACTTTGCCCCCTCATGTAAG-'3; CD2AP: forward: 5'-AAGGGTGGCTGGAAGGAGAAC-'3, reverse: 5'-ATGCCTTTCCCGTTTGATGG-' 3 . For miR quantification, the total RNA was isolated with an miRVana miRNA Isolation Kit, and TaqMan-based detection primers miR193a and U6 (internal control) (Thermo Fisher Scientific) were used for real-time amplification (ABI-7500, Applied Biosystems, Foster City, CA). Relative quantification of gene expression was calculated using the $\Delta \Delta C T$ method.

\section{Western Blot Analysis and Antibodies}

Protein blots of control and experimental cells were performed as previously described. ${ }^{4,29}$ Briefly, protein lysates were prepared using radioimmunoprecipitation assay lysis buffer (Millipore, Burlington, MA). After heating for 15 minutes, an equal volume of cell lysates was electrophoresed using $10 \%$ to $12 \%$ of SDS-PAGE gels. Separated proteins were transferred to polyvinylidene difluoride (EMD Millipore, Toronto, ON, Canada) membranes and processed further for immunostaining with primary antibodies against APOL1 (1G12D11; 1.57 $\mu \mathrm{g} / \mathrm{mL}$; mouse monoclonal, catalog number 661241-LG; ProteinTech), WT1 (C-19; $0.2 \mu \mathrm{g} / \mathrm{mL}$; catalog number SC192; rabbit polyclonal, Santa Cruz Biotechnology), synaptopodin $(\mathrm{P}-19 ; 0.2 \mu \mathrm{g} / \mathrm{mL}$; catalog number $\mathrm{SC} 21537$; rabbit polyclonal, Santa Cruz), CD2AP (B-4; $0.2 \mu \mathrm{g} / \mathrm{mL}$; catalog number SC25272; mouse monoclonal, Santa Cruz), podocalyxin $(0.2 \mu \mathrm{g} / \mathrm{mL}$; catalog number PAI-46170; rabbit polyclonal, Life Techologies), $\boldsymbol{\alpha}$-actinin $(\mathrm{H}-2 ; 0.2 \mu \mathrm{g} / \mathrm{mL}$; catalog number SC17829; mouse monoclonal, Santa Cruz), PAX2 $(0.29 \mu \mathrm{g} / \mathrm{mL}$; catalog number ab23799; rabbit polyclonal, Abcam), and claudin $1(0.35 \mu \mathrm{g} / \mathrm{mL}$; catalog number ab15078; rabbit polyclonal, Abcam). The blots were incubated with primary antibodies at $4{ }^{\circ} \mathrm{C}$ overnight and subsequently with horseradish peroxidase-labeled appropriate secondary antibodies (1:3000, Santa Cruz). The blots were developed using a chemiluminescence detection kit (Pierce, Rockford, IL) and exposed to X-ray film (Eastman Kodak, Rochester, NY) or chemiluminescence reader and Image Lab Software version 5.2.1 (Chemidoc MP, Bio-Rad
Laboratories). GAPDH (mouse monoclonal; 0411; $0.1 \mu \mathrm{g} /$ mL, catalog number SC47724; Santa Cruz Biotechnology) was used as an internal control to confirm equal protein loading.

\section{Luciferase Assay}

Complimentary APOL1 binding sites of miR193a were mapped by bioinformatics analysis using three different miRNA databases (www.mirbase.org, www.mirdb.org, and http://www.targetscan.org/vert_72; last accessed February 11, 2018). The APOL1 wild-type $3^{\prime}$ untranslated region (UTR) (pEZX-MT06-APOL1, HmiT055026-MT06) and pEZX-MT06-Control (scrambled nonspecific sequence $3^{\prime}$ UTR control vectors, CmiT000001-MT06) containing firefly luciferase and Renilla luciferase as tracking genes were purchased from GeneCopoeia Inc. (Rockville, MD). A Luc-Pair Duo-Luciferase High-Sensitivity Assay Kit was used to measure the relative luciferase activity as described in the manufacturer's protocols (GeneCopoeia).

Briefly, cells were transiently co-transfected by using Lipofectamine 2000 with wild-type or control reporter $3^{\prime}$ UTR plasmids and miR-193a (pCMV-miR-193a) or negative miR (control, AM17110) in combination. After 48 hours of co-transfection, the firefly luciferase activities were measured using the Duo-Luciferase HS Assay (GeneCopoeia). The relative luciferase activity was calculated by normalizing to Renilla luciferase. Results represent cumulative values of three independent experiments, each conducted in triplicates.

\section{Statistical Analysis}

Statistical comparisons were performed with the program PRISM using the $U$-test for nonparametric data and the unpaired $t$-test for parametric data. $P<0.05$ was accepted as statistically significant.

\section{Results}

\section{Generation of APOL1 Transgenic Mice}

A doxycycline-inducible APOL1 construct was generated by using plasmid pENTR3C as the backbone (Figure 1A). The cDNA of TetOn3G and its promoter PTRE3G were used from the Tet-On 3G Tetracycline-Inducible Expression System. The working flow chart is shown in Figure 1B. The purified construct DNA was introduced into the pronuclei of fertilized oocytes (from the FVB/N mouse) by microinjection using standard techniques.

To determine the time course effect, 4-week-old APOL1 (G1) transgenic mice $(n=3)$ were administered doxycycline $(2.5 \mathrm{mg} / \mathrm{kg}$ body weight daily for 3 consecutive days and then every other day) for different periods $(0,3,9$, and 18 days).

Subsequently, renal tissues were harvested. Proteins and RNAs were extracted. Protein blots were probed for APOL1 
and reprobed for GAPDH. cDNAs were amplified for APOL1 mRNA. Renal tissues showed APOL1 protein and mRNA (Figure 1C) expressions in a time course manner.

To evaluate the dose-response effect, 4-week-old APOL1 (G1) transgenic mice ( $n=3)$ were administered different doses of doxycycline intraperitoneally for 3 consecutive days.
Subsequently, renal tissues were harvested, and proteins and RNAs were extracted. Protein blots were probed for APOL1 and reprobed for GAPDH. cDNAs were amplified for APOL1 mRNA. Representative gels are shown in Figure 1D. Doxycycline enhanced APOL1 protein and mRNA expressions in a dose-dependent manner (Figure 1D).
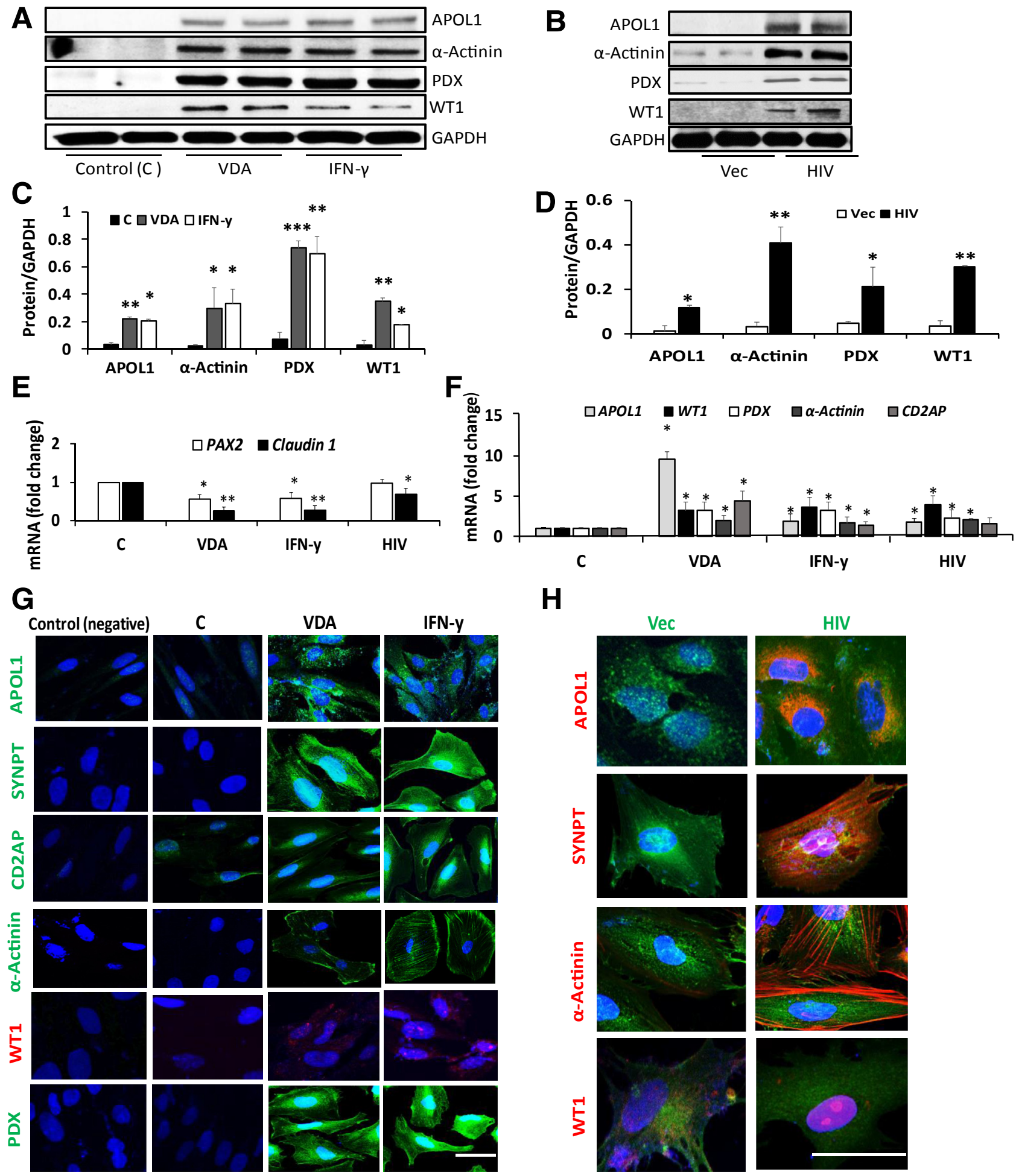

H
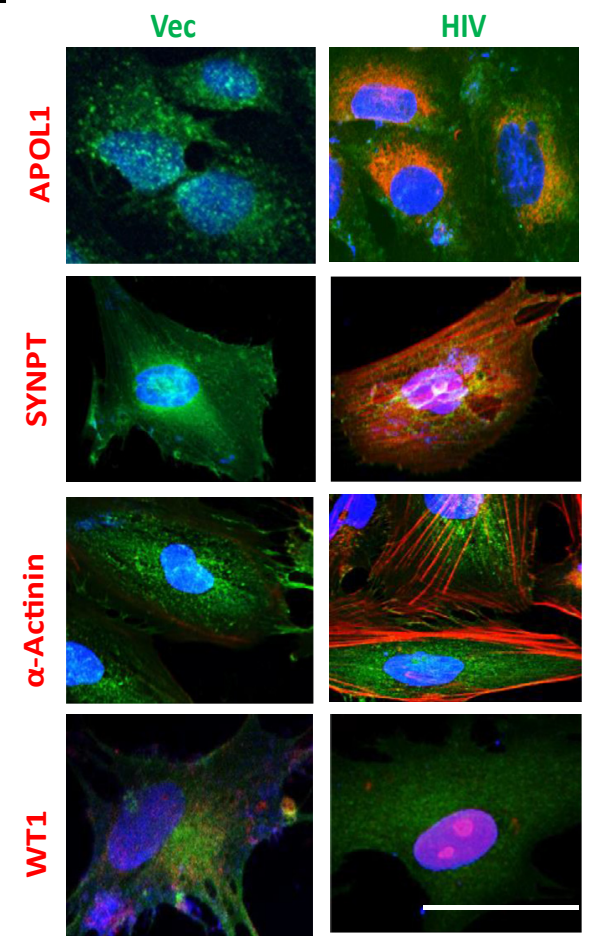


\section{AP0L1 and miR193a Are Reciprocally Linked with a} Feedback Loop in PECs

Human PECs do not display expression of APOL1 in vivo. ${ }^{1-3}$ To examine the expression of APOL1, lysates of cultured PECs (undifferentiated), differentiated podocytes, and HepG2 cells (display robust APOL1 protein expression; positive control) and HEK cells (kidney cells with undetectable APOL1 protein expression; negative control) were probed for APOL1 protein and GAPDH. HepG2 cells and podocytes displayed robust expression of APOL1, but PECs and HEK cells did not show any expression of the APOL1 protein (Figure 2A). These findings confirm that PECs do not express APOL1 protein expression in vitro either.

Both PECs and podocytes originate from the same mesenchymal cells during embryogenesis. ${ }^{20,21}$ However, only podocytes express APOL1..$^{1-4,30-33}$ It was tested whether PECs transiting to podocyte phenotype would also display APOL1. For differentiation of PECs, cells were incubated in the media described in Material and Methods for different intervals followed by RNA and protein extractions. Protein blots were probed for APOL1 and GAPDH (Figure 2B). PECs did not show any expression of the APOL1 protein on day 0. However, APOL1 expression appeared on day 4, and its expression increased further during the transition. These findings indicate that APOL1 protein expression emerges during the differentiation of PECs. RNAs were assayed for miR193a, and cDNAs were amplified with an $A P O L 1$-specific primer. Cumulative data are shown in Figure 2, C and D. There was an increase in APOL1 mRNA expression (Figure 2C) but a decrease in miR193a levels (Figure 2D) during the transition.

To examine a possible causal relationship, PECs (undifferentiated) were transfected with scrambled or APOL1 siRNAs. cDNAs were amplified for APOL1, and RNAs were quantified for miR193a. Cumulative data are shown in Figure 2E. Down-regulation of APOL1 mRNA levels was associated with the up-regulation of miR193a levels (Figure 2E). These results suggest that lack of APOL1 mRNA further enhances expression of miR193a, a marker of PEC phenotype. These findings further suggest that lack of APOL1 optimizes PEC phenotype.

To evaluate whether non-kidney cells expressing APOL1 also have a reciprocal link with miR193a, RNAs were extracted from control HepG2 cells, HepG2 cells transfected with scrambled siRNA, or APOL1 siRNA. RNAs were assayed for miR193a and APOL1 mRNA. Cumulative data are shown in Figure $2 \mathrm{~F}$. Down-regulation of APOL1 expression was associated with up-regulation of miR193a expression (Figure 2F). These findings suggest that APOL1 has an inverse relationship with mir193a expression in HepG2 cells.

To confirm an inverse relationship between APOL1 and miR193a in PECs, undifferentiated PECs were transduced with vector or APOL1 (lentivirus). Protein and RNAs were extracted from control, vector, and APOL1-transduced PECs. cDNAs were amplified for APOL1, and RNAs were quantified for miR193a. Cumulative data are shown in Figure 2G. An enhanced APOL1 expression was associated with down-regulation of miR193a expression (Figure 2G).

Interestingly, vector (lentivirus) also down-regulated miR193a expression when compared with control, but this effect was much lower when compared with APOL1induced miR193a levels. One possibility is that dsRNA activated interferon (IFN)- $\gamma$ in vector-transduced cells, which in turn resulted in decrease in miR193a expression.

If APOL1 is inversely regulating miR193a expression, it was determined whether miR193a may also reciprocally regulate APOL1 gene expression. Under such a scenario, down-regulation of miR193a should also derepress APOL1 expression in PECs. To achieve down-regulation of miR193a, PECs (undifferentiated) were transfected with empty vector (EV) or a specific miR193a inhibitor. Protein blots were probed for APOL1 and reprobed for GAPDH.

\footnotetext{
Figure 5 HIV, interferon (IFN)- $\gamma$, and vitamin D receptor (VDR) agonists induce apolipoprotein (APO) L1 and transition markers in parietal epithelial cells (PECS). A: To examine the effect of APOL1 stimulators on APOL1 induction and expression of PEC transition markers, PECs were incubated in media that contained vehicle [control (C)], VDR agonists (EB1089, $100 \mathrm{nmol} / \mathrm{L})$, or IFN- $\gamma(10 \mathrm{ng} / \mathrm{mL})$ for 48 hours. Protein blots were probed for APOL1, Wilms' tumor 1 (WT1), podocalyxin (PDX), and $\alpha$-actinin and reprobed for glyceraldehyde-3-phosphate dehydrogenase (GAPDH). Gels from two different lysates are shown. B: To assess the effect of HIV on APOL1 induction and expression of PEC transition markers, PECs were transduced with vector (Vec) or HIV [NL4-3, 10 ${ }^{3}$ green fluorescent protein (GFP)-expressing units (GEU)/mL]. Protein blots were probed for APOL1, WT1, podocalyxin, and $\alpha$-actinin and reprobed for GAPDH. Gels from two different lysates are shown. C: Cumulative densitometric data from the cells treated with VDA and IFN- $\gamma$ are shown. D: Cumulative densitometric data from the cells transduced with Vec or HIV are displayed. IFN- $\gamma-$, VDA receptor-, and HIV-induced APOL1 expression is associated with the expression of transition markers in PECs. E: To evaluate the effect of APOL1 induction on the transcription of PEC markers, RNAs were extracted from the lysates of HIV-, IFN$\gamma-$, and VDR agonist-treated cells (A and $\mathbf{B})$. cDNAs were amplified with specific primers for PAX2 and claudin 1. Cumulative data are shown in a bar diagram. APOL1 induction in PECs attenuates the expression of PEC markers. F: To examine the effect of APOL1 induction on the transcription of PEC transition markers, RNAs were extracted from the lysates of HIV-, IFN- $\gamma-$, and VDR agonist--treated cells (A and B). CDNA was amplified with specific primers for APOL1, WT1, $\alpha-$ actinin, PDX, and CD2AP. Cumulative data are shown in bar graphs. APOL1 induction in PECs results in enhanced transcription of PEC transition markers. G: To visualize the expression of PEC transition markers in response to APOL1 inducers, PECs grown on coverslips were treated under similar conditions (as in A) and labeled for PEC transition markers. Representative fluoromicrographs are displayed. Expression of APOL1, $\alpha$-actinin, and PDX is indicated by green fluorescence and of WT1 by red fluorescence. H: To visualize the expression of PEC transition markers in response to HIV, PECs grown on coverslips were transduced with VEC (GFP positive) or HIV (GFP positive) and labeled for PEC transition markers. Representative fluoromicrographs are displayed. Both Vec- and HIV-transduced cells are GFP positive (indicated by green fluorescence). HIV-transduced cells display an overt expression of APOL1, synaptopodin (SYNPT), $\alpha$-actinin, and WT1 (red fluorescence). $n=4(\mathbf{A}$ and $\mathbf{B}) ; n=3(\mathbf{E}$ and $\mathbf{F}) .{ }^{*} P<0.05,{ }^{* *} P<0.01,{ }^{* *} P<0.001$ versus respective control. Scale bars: $5 \mu \mathrm{m}(\mathbf{G}$, all panels); $10 \mu \mathrm{m}$ (H, all panels). VDA, VDR agonist.
} 

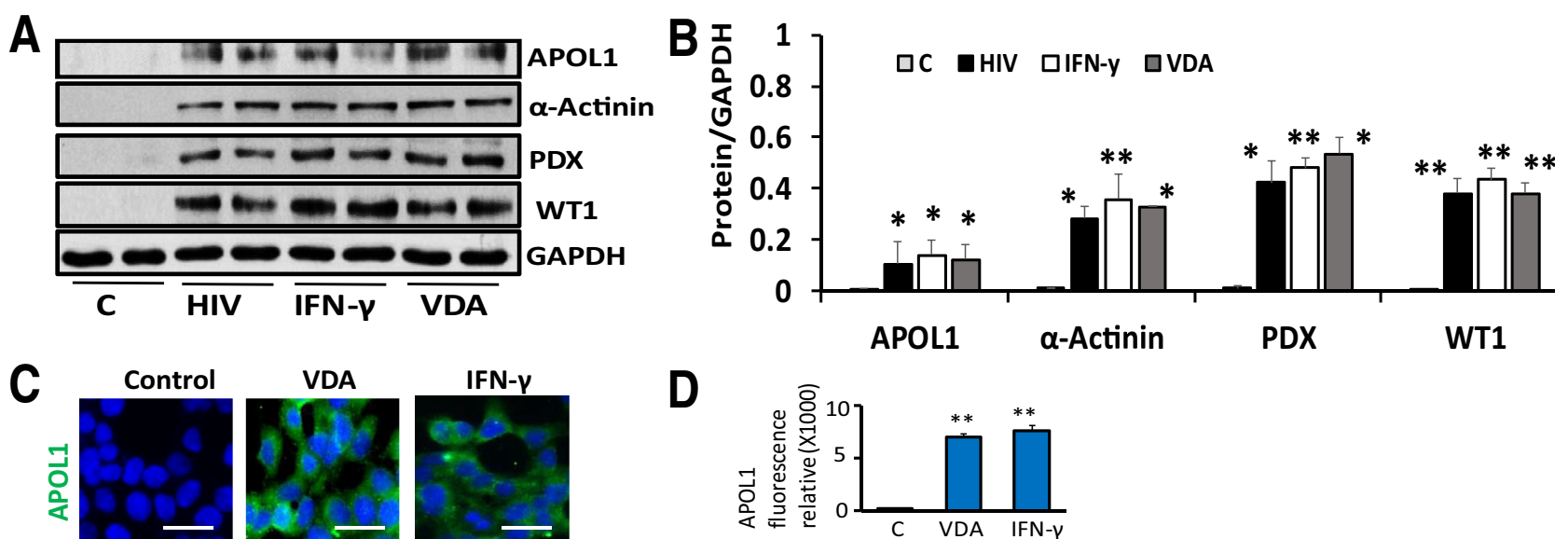

D
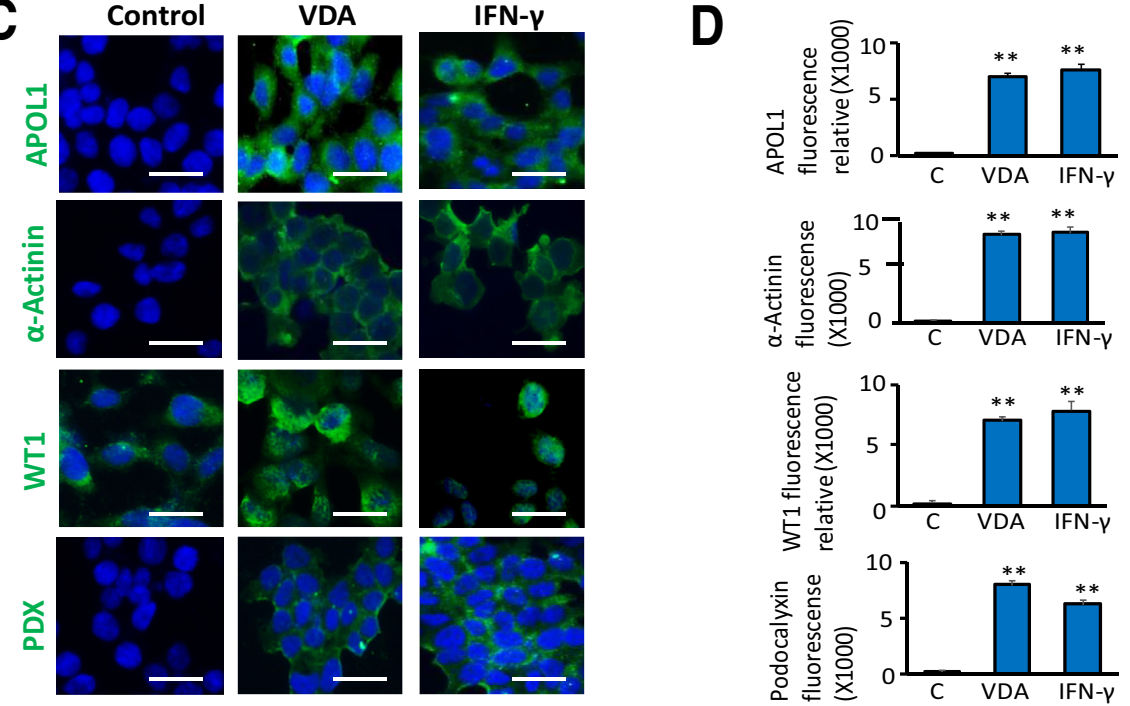

Figure 6 HIV, interferon (IFN)- $\gamma$, and a vitamin D receptor (VDR) agonist induce apolipoprotein (APO) L1 and transition markers in human embryonic kidney (HEK) cells. A: To examine the effect of APOL1 stimulators on the induction of APOL1 and associated downstream signaling in kidney cells with undetectable APOL1 expression, HEK cells were transduced with HIV (NL4-3, $10^{3}$ green fluorescent protein-expressing units/mL) or treated with IFN- $\gamma$ (10 ng/ $\mathrm{mL}$ ) or a VDR agonist (EB1089, $100 \mathrm{nmol} / \mathrm{L})$ for 48 hours. Protein blots were probed for AP0L1, Wilms' tumor 1 (WT1), podocalyxin (PDX), and $\alpha$-actinin and reprobed for glyceraldehyde-3-phosphate dehydrogenase (GAPDH). Representative gels from two different lysates are shown. B: Cumulative densitometric data from the protein blots of lysates from $\mathbf{A}$ are shown in a bar diagram. APOL1 inducers enhance the expression of parietal epithelial cell (PEC) transition markers in HEK cells. C: To visualize the downstream effects of APOL1 inducers in kidney cells with undetectable APOL1 expression, HEK cells grown on coverslips were treated with IFN- $\gamma(10 \mathrm{ng} / \mathrm{mL})$ or VDR agonist $(100 \mathrm{nmol} / \mathrm{L})$ for 48 hours. Subsequently, cells were labeled for APOL1, PDX, $\alpha$-actinin, and WT1 and examined under a confocal microscope. Representative fluoromicrographs are displayed. VDR agonist and IFN- $\gamma$ induce the expression APOL1, PDX, $\alpha$-actinin, and WT1 (indicated by green fluorescence) in HEK cells. D: Quantification of fluorescence data (25 to 40 cells in each group) from C is shown in bar graphs. B and D: APOL1 stimulators induce the expression of transition markers in HEK cells. $n=4(\mathbf{A}) ; n=3$ (B). ${ }^{*} P<0.05,{ }^{*} P<0.01$, versus respective control. Scale bars $=2.5 \mu \mathrm{m}(\mathbf{C})$. VDA, VDR agonist.

Blots of three different lysates of control and experimental PECs are shown in Figure 2H. miR193a inhibitor induced the expression of the APOL1 protein in PECs. These findings indicate that miR193a also inversely regulates APOL1 expression in PECs.

To determine the dose-response effect of miR193a inhibition on the APOL1 transcription, undifferentiated PECs were incubated in media that contained different concentrations of miR193a inhibitor for 48 hours. Subsequently, RNAs were extracted. RNAs were assayed for miR193a and APOL1 mRNA. Cumulative data are shown in Figure 2I. Inhibition of miR193a resulted in enhanced expression of APOL1 in a concentration-dependent manner.

To evaluate the time course effect of miR193a inhibition on APOL1's transcription, PECs were incubated in media that contained a specific inhibitor of miR193a for different periods and evaluated for APOL1 mRNA expression. Cumulative data are shown in Figure 2J. Inhibition of miR193a expression enhanced APOL1 mRNA expression in PECs in a time-dependent manner.

To confirm a reciprocal relationship between miR193a and APOL1 expression in HepG2 cells, cells were transfected with EV or different doses of miR193a plasmids. cDNAs were amplified for APOL1 mRNA, and RNAs were assessed for miR193a. Cumulative data are shown in Figure 2K. Enhanced miR193a expression in HepG2 cells was associated with down-regulation of APOLl expression (Figure 2K).

These results show that APOL1 and miR193a are reciprocally linked with a feedback relationship in human PECs. This relationship is also present in non-kidney cells, such as HepG2 cells, which express APOL1. 
A

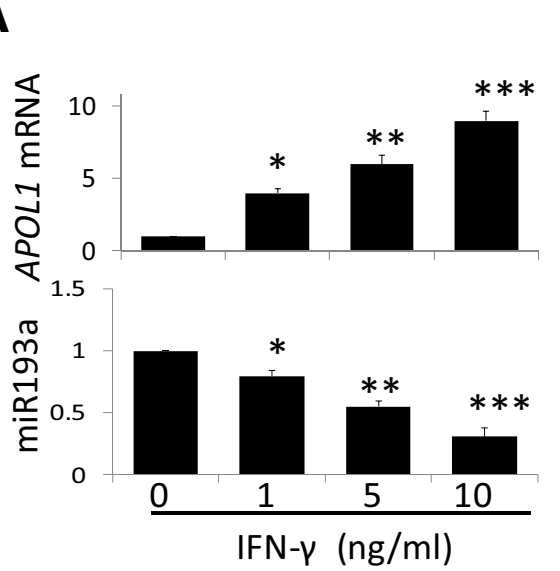

B

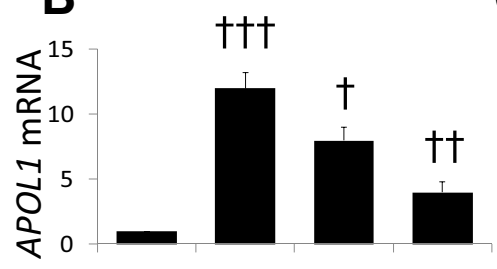

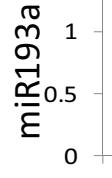

$0+$

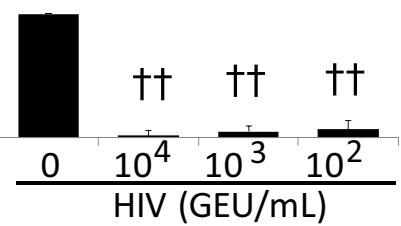

C

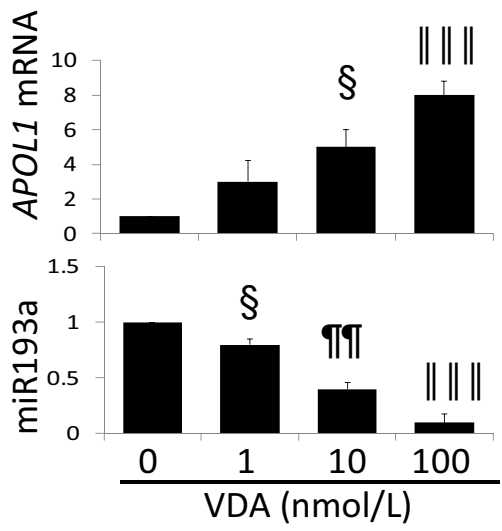

D

Claudin 1 PAX2
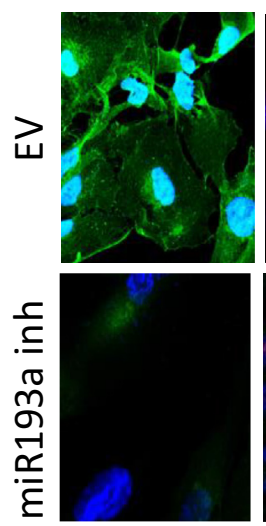

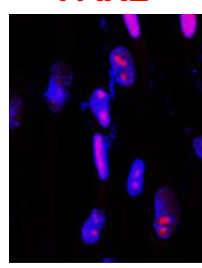

APOL1
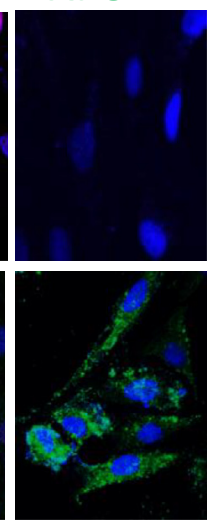

a-Actinin
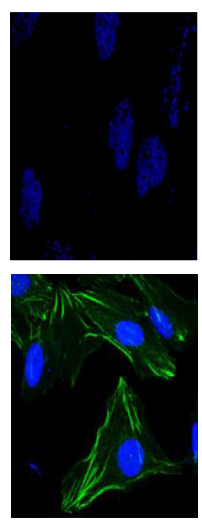

WT1

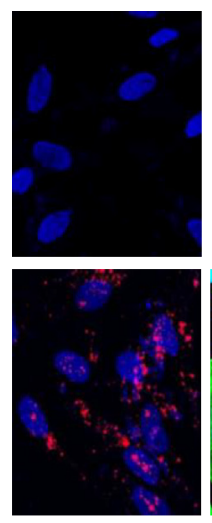

PDX
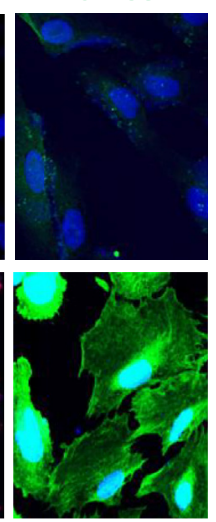

SYNPT
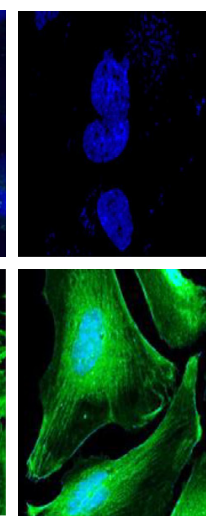

\section{CD2AP}
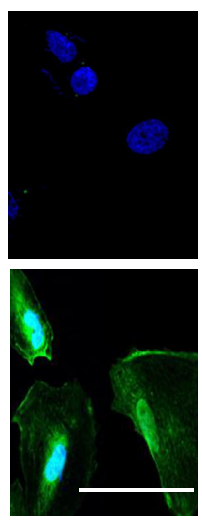

Figure 7 Role of miR193a in HIV-, interferon (IFN)- $\gamma-$, and vitamin D receptor (VDR) agonist-mediated APOL1 induction in parietal epithelial cells (PECs). A: To examine the role of miR193a in the induction of APOL1 by IFN- $\gamma$, PECs were incubated in media that contained a different concentration of IFN- $\gamma(0,1,5$, and $10 \mathrm{ng} / \mathrm{mL})$ for 48 hours and assayed for miR193a. CDNAs were amplified with a primer specific for APOL1. Cumulative data on the expression of miR193a and APOL1 mRNA are shown in the top row and bottom row, respectively. IFN- $\gamma$ down-regulates miR193a expression and enhances the transcription of APOL1 in a dose-dependent manner. B: To assess the role of miR193a in the induction of APOL1 by HIV, PECs were transduced with different concentrations of HIV [NL4-3; $10^{4}, 10^{3}$, or $10^{2}$ green fluorescent protein-expressing units (GEU)/mL]. After 48 hours, RNAs were assayed for miR193a. cDNAs were amplified for APOL1. Levels of miR193a and APOL1 mRNA are shown in the top row and bottom row, respectively. HIV decreases the expression of miR193a and enhances the transcription of APOL1 in a dose-dependent manner. C: To evaluate the role of miR193a in the induction of APOL1 by VDR agonist, PECs were incubated in media that contained different concentrations of VDR agonist (EB1089, 0, 1, 10, and 100 nmol/L; 0 containing the vehicle only) for 48 hours. RNAs were assayed for miR193a. cDNAs were amplified for APOL1. Cumulative data on the expression of miR193a and APOL1 mRNA are shown in the bottom row and top row, respectively. VDR agonist attenuated miR193a levels but enhances the expression of APOL1 mRNA in a dose-dependent manner. D: To validate the effect of miR193a inhibition on the expression of PECs and PEC transition markers, PECs (undifferentiated) grown on coverslip were transfected with an empty vector (EV) or inhibitor of miR93a ( $50 \mathrm{nmol} / \mathrm{L}$ ). Subsequently (after 48 hours), cells were labeled for PECs and PEC transition markers. Cells were examined under a confocal microscope. Representative fluoromicrographs are displayed. Inhibition of miR193a down-regulates PEC markers but enhances the expression of transition markers in PECs. $n=3 .{ }^{*} P<0.05, * * * P<0.001$ versus respective $0 \mathrm{ng} / \mathrm{mL}$ of IFN$\gamma ;{ }^{* \star} P<0.01$ versus respective $1.0 \mathrm{ng} / \mathrm{mL}$ of IFN- $\gamma ;{ }^{\dagger} P<0.05$ versus $0,10^{4}$ and $10^{2} \mathrm{HIV} ;{ }^{\dagger \dagger} P<0.01,{ }^{\dagger \dagger \dagger} P<0.001$ versus respective 0 HIV; ${ }^{\ddagger} P<0.05$ versus 0 and $100 \mathrm{nmol} / \mathrm{L}$ VDR agonist; ${ }^{\ddagger \ddagger} P<0.01$ versus respective $0,1.0$, and $100 \mathrm{nmol} / \mathrm{L}$ VDR agonist; ${ }^{\ddagger \ddagger \ddagger} P<0.001$ versus respective 0 . Scale bar $=50$ $\mu \mathrm{m}$ (D, all panels). VDA, VDR agonist.

\section{APOL1 Expression Is Associated with the Expression of PEC Transition Markers}

We have previously reported the characteristics of cultured PECs in undifferentiated ( 0 days) and differentiated states (14 days). ${ }^{20}$ To characterize PEC molecular phenotype further, proteins and RNAs were extracted from control (cultured at $33^{\circ} \mathrm{C}$; undifferentiated) and transited (differentiated) PECs (PECs seeded on fibrin-coated plates and incubated in special media for 14 days at $37^{\circ} \mathrm{C}$ ). Subsequently, proteins and RNAs were extracted. Protein blots were probed for several PEC and podocyte markers and reprobed for GAPDH $(n=4)$ ). Representative gels (on 0 and 14 days) are displayed in Figure 3, A and C. Densitometric data are shown in Figure 3, B and D. PEC transition was characterized by down-regulation of PEC markers (PAX2 and claudin 1) (Figure 3, A and B), induction of APOL1 (Figure 3, C and D), and up-regulation of transition 
A

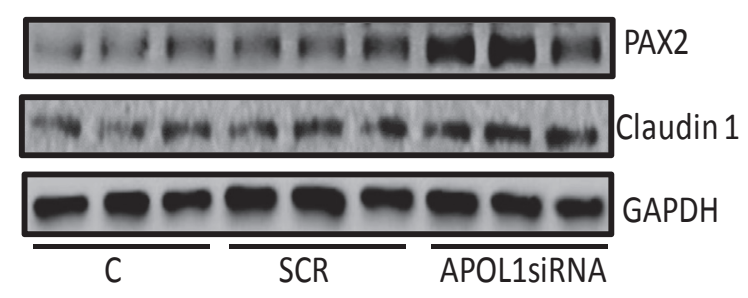

C
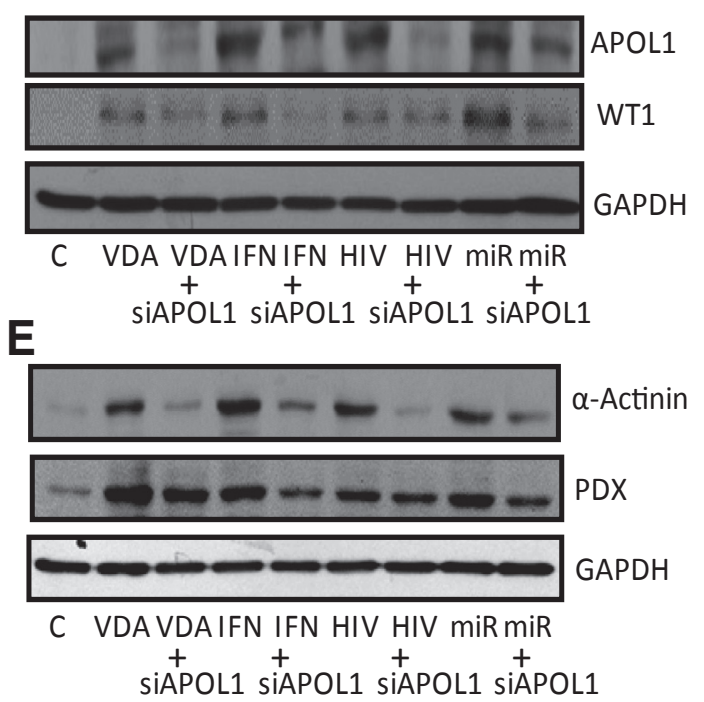

B
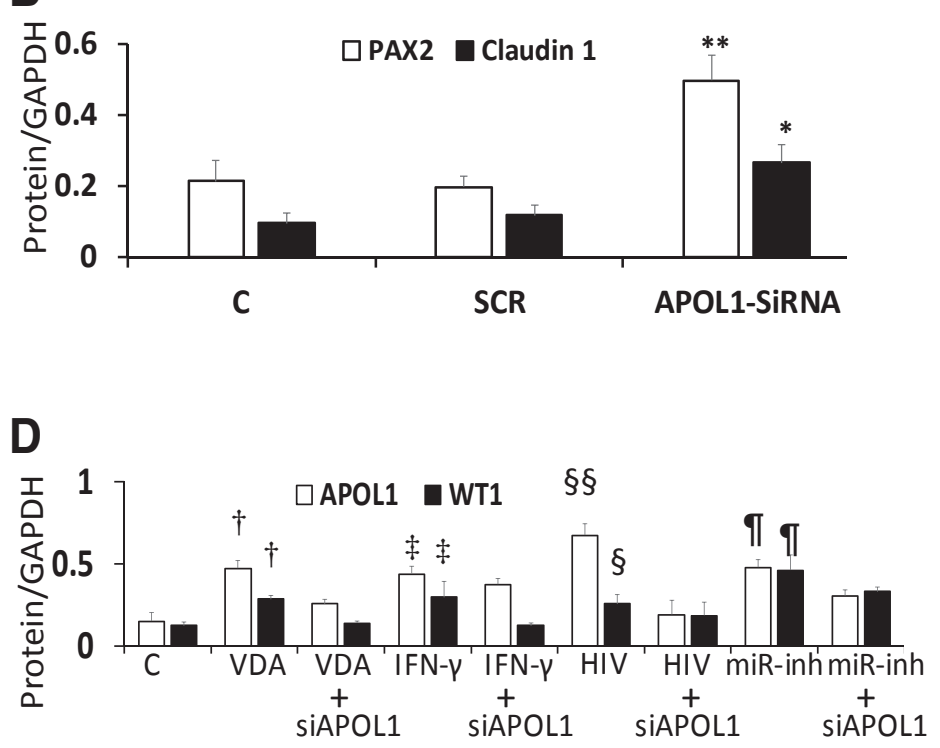

$\mathbf{F}$

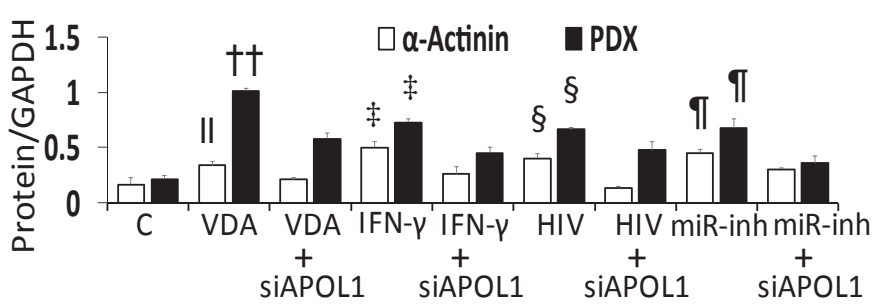

Figure 8 Apolipoprotein (APO) L1 is critical for the functionality of APOL1-miR193a axis. A: To evaluate the effect of APOL1 silencing on parietal epithelial cell (PEC) markers, PECs (undifferentiated) were transfected with scrambled (SCR) or APOL1 siRNAs. Protein blots control cells and transfected cells were probed for PAX2, claudin 1, and glyceraldehyde-3-phosphate dehydrogenase (GAPDH). Gels from three different lysate preparations are displayed. B: Cumulative densitometric data from the above-mentioned lysates are shown in a bar diagram. APOL1 silencing in PECS with undetectable APOL1 protein enhances the expression of PEC markers. C: To assess whether APOL1 is critical for the expression of PEC transition markers, PECs were transfected with SCR or APOL1 siRNA. Control (C) and transfected cells were incubated in media that contained vehicle, vitamin D receptor (VDR) agonist (100 nmol/L), interferon (IFN)- $\gamma(10 \mathrm{ng} / \mathrm{mL}$ ), or miR193a inhibitor (miR, $50 \mathrm{nmol} / \mathrm{L})$ for 48 hours. In parallel sets of experiments, $C$ and transfected cells were transduced with HIV (NL4-3; $10^{3}$ green fluorescent protein-expressing units/mL). Protein blots were probed for APOL1 and reprobed for Wilms' tumor 1 (WT1) and GAPDH. Representative protein gel blots are displayed. D: Cumulative densitometric data of protein blots from C are shown in bar graphs. E: Protein gel blots from the cellular lysates used in $8 \mathrm{C}$ were probed for $\alpha$-actinin, podocalyxin (PDX), and GAPDH. Representative blots are displayed. F: Cumulative densitometric data of protein blots from $\mathbf{E}$ are shown in a bar diagram. $n=4 .{ }^{*} P<0.05,{ }^{* *} P<0.01$ versus respective control and SCR $(\mathbf{B})$; ${ }^{\dagger} P<0.05$, ${ }^{\dagger} P<0.01$ versus respective $C$ and VDR agonist + siAPOL1; ${ }^{\ddagger} P<0.05$ versus respective $C$ and IFN-Y + siAPOL1; ${ }^{\S} P<0.05$ versus respective $C$ and HIV and siAPOL1; ${ }^{\S \S} P<0.01$ versus respective $C$ and HIV + siAPOL1; ${ }^{\top} P<0.05$ versus respective $C$ and miR193a inh + siAPOL1 (D and F); $\| P<0.05$ versus respective $C(\mathbf{F})$. VDA, VDR agonist.

(podocyte differentiation) markers (Figure 3, C and D). cDNAs were amplified with specific primers for PECs and their transition markers. Cumulative data are shown in Figure 3, E and F. PEC transition was associated with down-regulation of the transcription of PAX2 and claudin 1 (Figure $3 \mathrm{E}$ ) and up-regulation of the transcription of transition markers (Figure 3F).

To determine the time course effect of loss and gain of PECs and transition markers, PECs (undifferentiated) were incubated for different periods at $37^{\circ} \mathrm{C}$. cDNAs were amplified for PEC transition markers, and cumulative data are shown in Figure 3, G and H. Transited PECs displayed a decrease in PEC but a several-fold increase in their transition markers along with APOL1 expression.

To visualize the alterations of loss of PEC markers and gain of differentiation markers during the transition of PECs, undifferentiated PECs were grown on coverslips. Undifferentiated (cultured at $33^{\circ} \mathrm{C}$ ) and transited (differentiated) PECs (PECs seeded on fibrin-coated plates and incubated in special media for 14 days at $37^{\circ} \mathrm{C}$ ) were labeled for PEC markers and transition markers. Representative fluoromicrographs are displayed, showing down-regulation of PEC markers but induction of APOL1 and transition markers (Figure 3I). In brief, the appearance of APOL1 is associated with down-regulation of PEC markers and up-regulation of transition markers. 

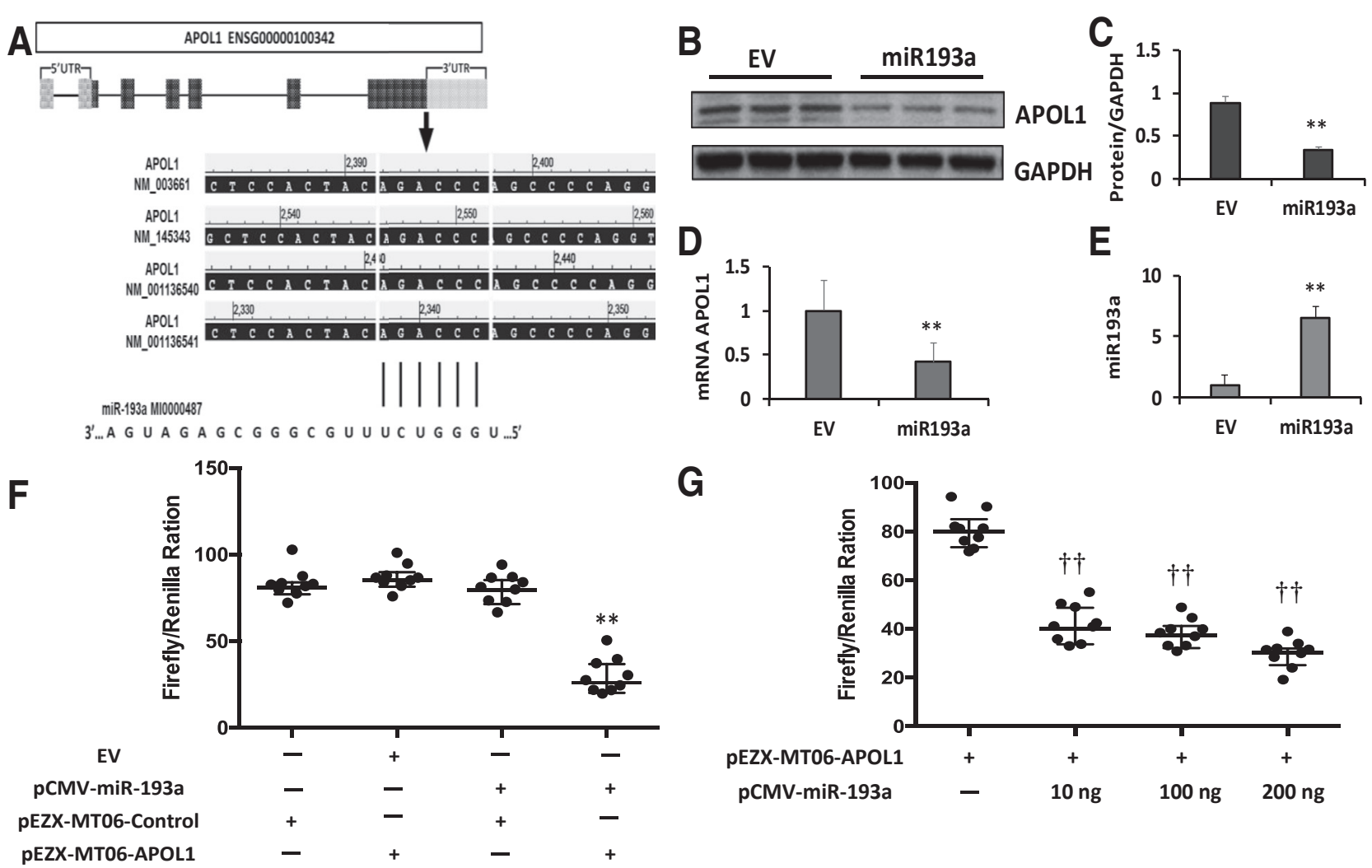

G

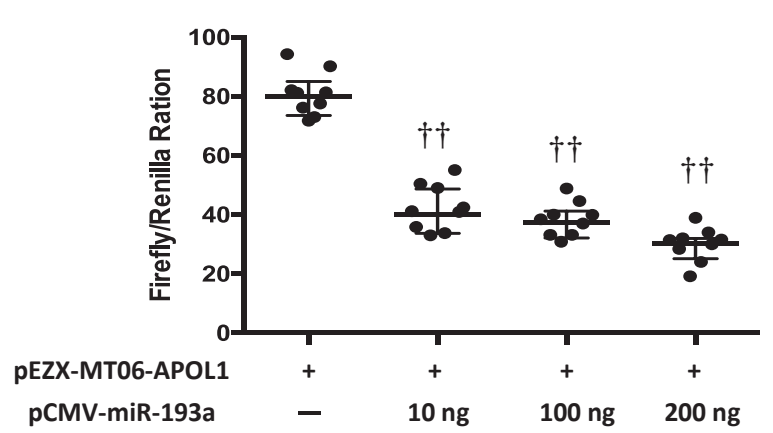

Figure 9 Apolipoprotein (APOL) 1 is a potential target for miR193a. A: In silico computational algorithms based on schematic representation of predicted miR193a target site in APOL1 3'-untranslated region. B: HepG2 cells were transiently transfected with empty vector (EV) or miR193a plasmids. Protein blots were probed for APOL1 and GAPDH. Gels from there different lysates are displayed. C: Cumulative data of protein blots shown in B are shown in bar graphs. D: RNAs were extracted from the lysates of B. cDNAs were assessed for APOL1 mRNA. Cumulative data are shown in a bar diagram. E: RNAs from the lysates of B were assayed for miR193a. Cumulative data are shown in a bar diagram. F: Human embryonic kidney (HEK) cells were transfected with EV, pcMV-miR193a, pEZX-MT06 (control), or pEZx-MT06-APOL1 and evaluated for APOL1 luciferase signaling. HEK cells overexpressing pCMV-miR193a display decreased pEZXMT06-APOL1 luciferase signal compared with EV. G: Relative luciferase activity with different concentrations of pCMV-miR193a. These findings indicate the presence of putative interaction between miR193a and APOL1. $n=3 .{ }^{* *} P<0.01$ versus $\mathrm{EV}^{{ }^{\dagger}}{ }^{\dagger} P<0.01$ versus 0 concentration.

\section{Induction of APOL1 Protein Expression in HEK Cells Induces the Expression of Transition Markers}

To test whether APOL1 is an inducer of transition markers, likely in other kidney cells (with undetectable APOL1 protein expression) as well, HEK cells were transfected with EV or APOL1 plasmids $(n=4)$. Subsequently, proteins and RNAs were extracted. Protein blots were assayed for APOL1 and reprobed for transition markers. Representative gels from two different lysates are displayed in Figure 4A. Cumulative densitometric data are shown in Figure 4B.

EV-transfected HEK cells did not show any expression of APOL1. On the contrary, APOL1-transfected HEK cells displayed moderate expression of transition markers (Figure 4, A and B). EV-transfected HEK cells displayed only a minimal expression of WT1. cDNAs were amplified for PEC markers and transition markers. Cumulative data are shown in Figure 4, C and D. APOL1 induction was associated with attenuated transcription of PAX2 and claudin 1 (Figure 4C). On the other hand, APOL1transfected HEK cells displayed an enhanced transcription of APOL1, WT1, podocalyxin, and CD2AP (Figure 4D). Thus, it appears that induction of APOL1 carries the potential to stimulate the expression of transition markers in HEK cells otherwise with undetectable APOL1 protein expression.

\section{HIV, IFN- $\gamma$, and VDR Agonists Induce APOL1 and Transition Markers in PECs}

Whether IFN- $\gamma$, vitamin D receptor (VDR) agonists, and HIV (known podocyte APOL1 stimulators ${ }^{4,28}$ ) carry a potential to induce APOL1 expression in PECs was examined. PECs (undifferentiated) were incubated in media that contained vehicle (control, DMSO), VDR agonists, or IFN- $\gamma$ for 48 hours $(n=4)$. In parallel sets of experiments $(n=4)$, PECs were also transduced with vector or HIV (NL4-3). Protein blots were probed for APOL1 and reprobed for transition markers. Gels from two different lysates are displayed in Figure 5, A and B. Cumulative densitometric data are shown in Figure 5, C and D. Control and vector-expressing PECs did not show any expression of the APOL1 protein. On the other hand, VDR 
agonists, IFN- $\gamma$, and HIV not only induced APOL1 expression in PECs but also stimulated the expression of PEC transition markers. RNAs were extracted from the above-noted lysates. cDNAs were amplified for PEC and transition makers $(n=3)$. Cumulative data are shown in Figure 5, E and F. HIV, IFN- $\gamma$, and VDR agonists decreased the transcription of claudin 1 . IFN- $\gamma$ and VDR agonists also decreased the transcription of $P A X 2$. VDR agonists and IFN $-\gamma$ not only increased the transcription of
APOL1 but also stimulated the transcription of transition markers. HIV increased the transcription of all the transition markers except $C D 2 A P$.

PECs (undifferentiated) grown on coverslips were treated as above (VDR agonists, IFN- $\gamma$, and HIV) and immunolabeled for different transition markers. Representative fluoromicrographs are shown in Figure 5, G and $\mathrm{H}$. IFN- $\gamma$, VDR agonists, and HIV induced the expression of APOL1 and PEC transition markers. In PECs as well, an
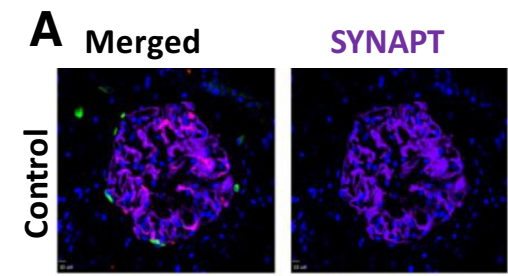

APOL1

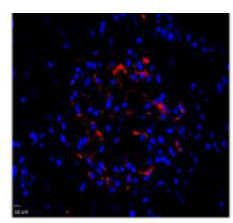

Cytok

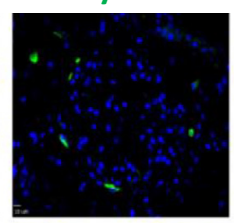

SYNAPT + Cytok Cytok + APOL1 SYNAPT + APOL1
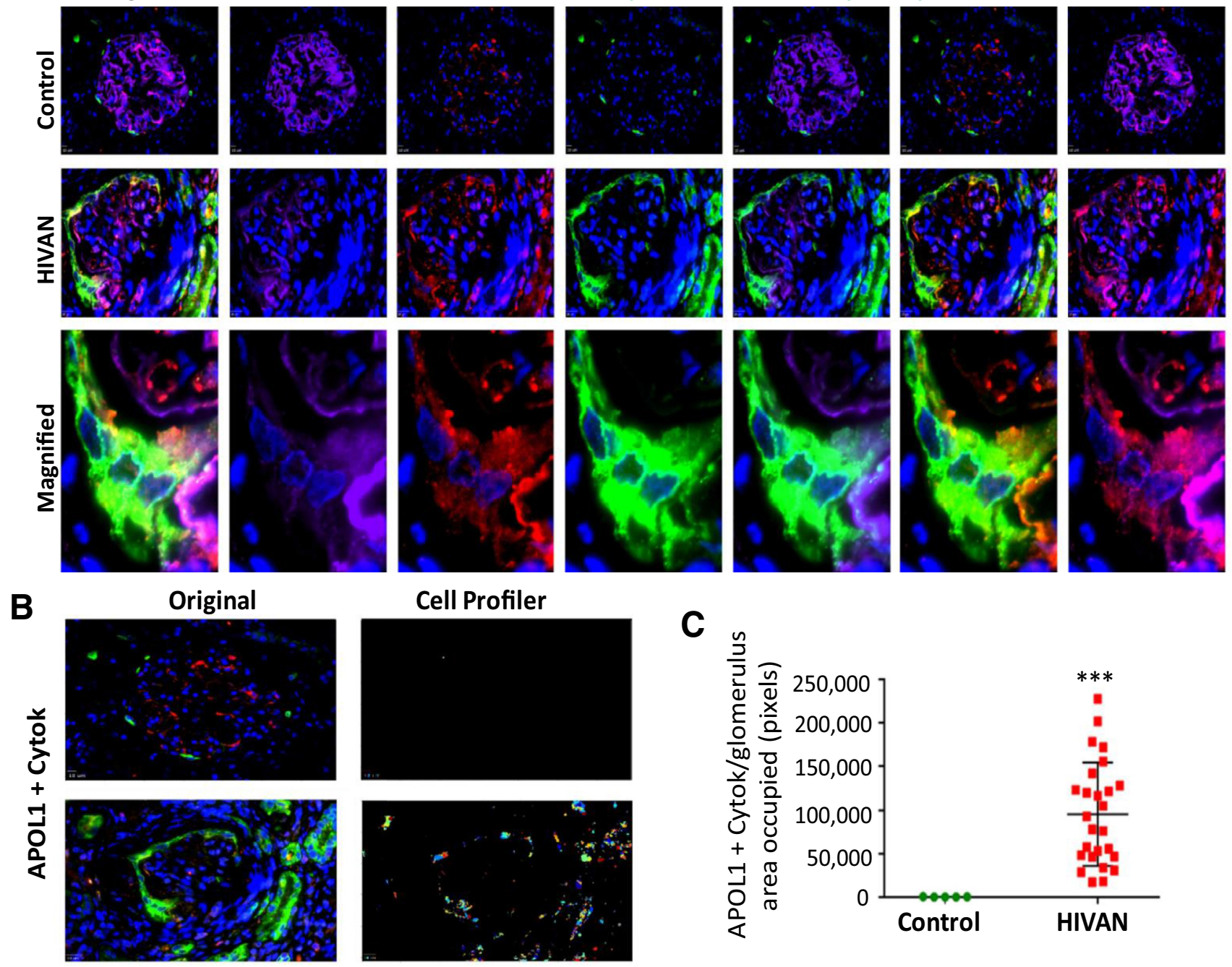

C
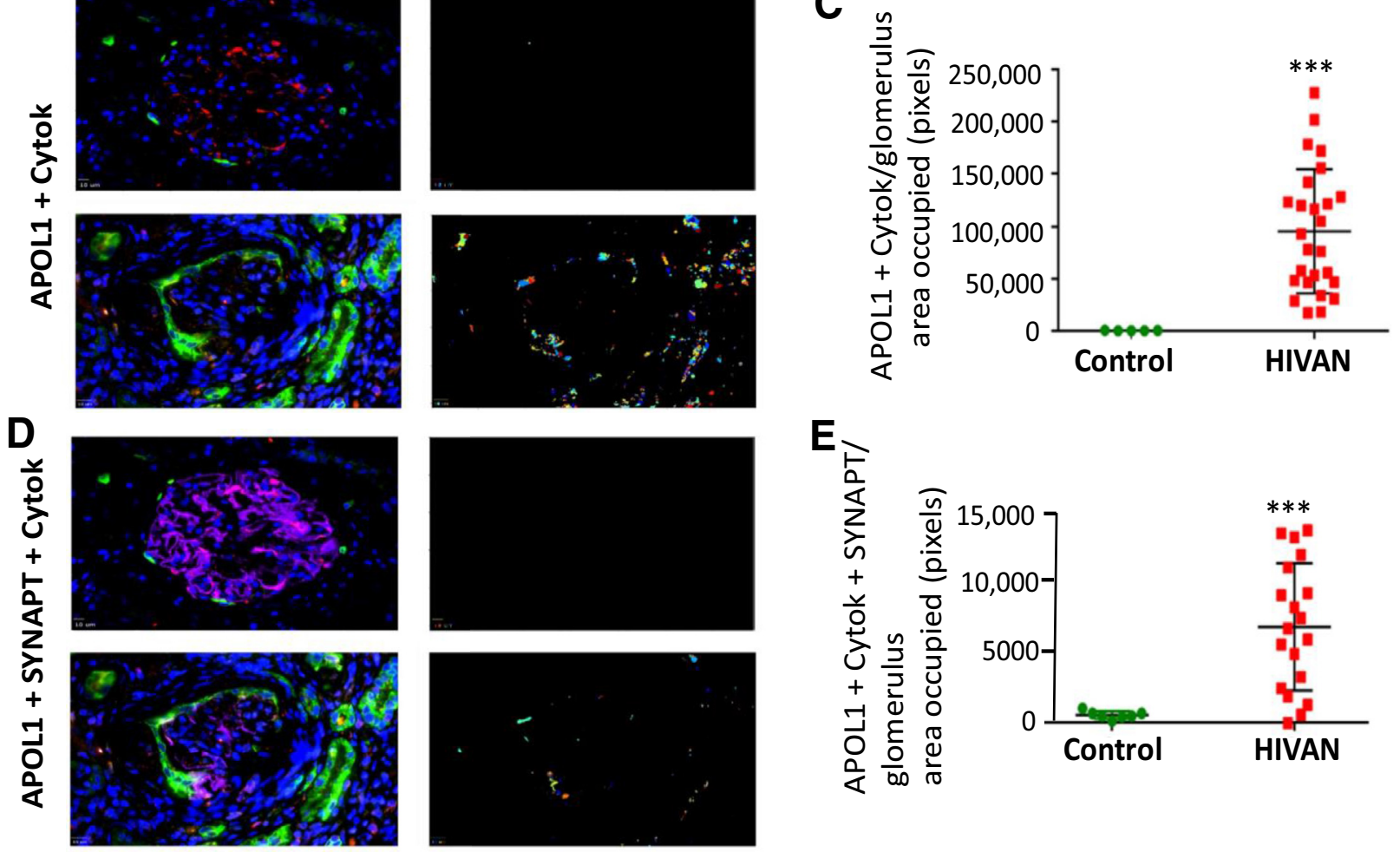
induction of APOL1 stimulates the expression of transition markers.

\section{HIV, IFN- $\gamma$, and VDR Agonists Induce APOL1 and Transition Markers in HEK Cells}

To test whether known podocyte APOL1 stimulators ${ }^{4,30,31}$ also carry a potential to induce APOL1 and associated downstream effects in kidney cells that lack detectable APOL1 protein expression, HEK cells were incubated in media that contained vehicle (control, DMSO), IFN- $\gamma$, or VDR agonists; in parallel sets of experiments $(n=4)$, HEK cells were transduced with HIV (N14-3; $10^{3}$ GEU/ml). Proteins were extracted. Blots probed for APOL1 and reprobed for transition markers. Gels from two different lysates are displayed in Figure 6A. Cumulative densitometric data are shown in Figure 6B. Control cells did not show any expression of APOL1 or transition markers. On the other hand, HIV, IFN- $\gamma$, and VDR agonists not only induced APOL1 expression in HEK cells but also induced the expression of transition markers. To visualize the expression of APOL1 and transition markers, HEK cells grown on coverslips were treated with vehicle (DMSO, control), VDR agonists, or IFN- $\gamma$ for 48 hours. Subsequently, cells were labeled for APOL1 and transition markers and examined under a confocal microscope. Representative fluoromicrographs are displayed in Figure 6C. Fluorescence quantification data are shown in Figure 6D. Interestingly, APOL1 stimulators induced not only APOL1 protein expression in HEK cells (cells with no detectable APOL1 protein) but also the expression of PEC transition markers.

\section{Role of miR193a in HIV-, IFN- $\gamma-$, and VDR Agonist-Mediated APOL1 Induction in PECs}

Although HIV, IFN- $\gamma$, and VDR agonists are known to
enhance APOL1 expression in podocytes, ${ }^{4,30,31}$ the mech-
anism is not known. Because miR193a inversely regulates
APOL1 transcription, we asked whether HIV-, IFN- $\gamma-$, and VDR agonist-induced APOL1 transcription is mediated through repression of miR193a expression.

To determine the dose-response effect of IFN- $\gamma$, PECs (undifferentiated) were treated with different doses of IFN- $\gamma$ for 48 hours. RNAs were extracted and assayed for miR193a and APOL1 mRNA. Cumulative data are shown in Figure 7A. IFN- $\gamma$ down-regulated PEC expression of miR193a in a dose-dependent manner (Figure 7A). However, IFN- $\gamma$ also enhanced the transcription of APOL1 in a dose-dependent manner. These findings suggest IFN- $\gamma$ may be enhancing the transcription of APOL1 through the repression of miR193a.

To confirm whether HIV is also using the same modus operandi to enhance transcription of APOL1, PECs were transduced with different concentrations of HIV (NL4-3; $10^{4}, 10^{3}$, or $\left.10^{2} \mathrm{GEU} / \mathrm{mL}\right)(n=3)$. Subsequently, RNAs were assessed for miR193a and APOL1 mRNA expressions. Cumulative data are shown in Figure 7B. HIV downregulated the expression of miR193a but up-regulated APOL1 mRNA expression (Figure 7B).

Because vitamin $\mathrm{D}$ has also been shown to down-regulate miR193a expression in PECs,${ }^{20}$ the effect of a VDR agonist was evaluated on APOL1 induction in PECs. PECs were treated with different concentrations of VDR agonist for 48 hours.

Subsequently, RNAs were extracted. RNAs were evaluated for miR193a and APOL1 mRNA. Cumulative data are shown in Figure 7C. VDA not only down-regulated miR193a expression but also enhanced APOL1 expression (Figure 7C).

If down-regulation of miR193a was inducing the expression of APOL1 in PECs, then inhibition of miR193a should not only induce APOL1 but should also stimulate the expression of transition markers. Moreover, if the enhanced expression of miR193a in PECs acts to sustain the PEC phenotype, then inhibition of miR193a should attenuate the expression of PEC markers. To evaluate the effect of miR193a inhibition, undifferentiated PECs grown on

\footnotetext{
Figure 10 Analysis of parietal epithelial cells (PECS) expressing apolipoprotein (APOL) 1 and synaptopodin (SYNAPT) in renal biopsy specimens of patients with HIV-associated nephropathy (HIVAN). A: Paraffin-fixed renal biopsy specimens of controls and patients with HIVAN co-labeled for cytokeratin (green fluorescence), synaptopodin (purple fluorescence), and APOL1 (red fluorescence). Representative fluoromicrographs are displayed. A glomerulus in a control patient does not show any expression of APOL1 by PECs, but a glomerulus in a patient with HIVAN displays APOL1 expression by PECs (yellow fluorescence in the co-labeled image). An occasional PEC also displays co-labeling for APOL1 and SYNAPT. B: A pipeline of modules used for the analysis of the PECs expressing APOL1 or APOL1 and cytokeratin (Cytok) using Broad Institute's CellProfiler suite. Representative original images of the glomeruli from controls and patients with HIVAN showing expression of APOL1 (red fluorescence) and Cytok (green fluorescence) were captured using SlideBook software version 6.0. Images were then processed using the CellProfiler pipeline to analyze PECs that expressed APOL1. The processed images are showing randomly denoted colors of the area occupied by pixels expressing Cytok and APOL1. C: More than 20 glomeruli from eight biopsy samples from patients with HIVAN were analyzed, and data of area occupied by pixels expressing Cytok and APOL1 were collected. A dot plot is showing the number of pixels co-expressing Cytok and APOL1 between controls and patients with HIVAN. A dot plot is showing a comparison of the number of pixels in the area occupied by PECs co-expressing Cytok and APOL1. D: A pipeline of modules used for the analysis of the PECs expressing APOL1 or APOL1 and Cytok using Broad Institute's CellProfiler suite. Representative original images of the glomeruli from controls and patients with HIVAN showing expression of APOL1 (red fluorescence), Cytok (green fluorescence), and SYNAPT (purple) were captured using SlideBook software version 6.0. Images were then processed using CellProfiler pipeline to analyze PECs expressing APOL1. The processed images are showing randomly denoted colors of the area occupied by pixels expressing Cytok and APOL1. E: More than 20 glomeruli from eight biopsy specimens from patients with HIVAN were analyzed, and data of the area occupied by pixels expressing Cytok, SYNAPT, and APOL1 were collected. A dot plot shows the number of pixels co-expressing Cytok, SYNAPT, and APOL1 between controls and patients with HIVAN. A dot plot shows a comparison of the number of pixels in the area occupied by PECs co-expressing Cytok, SYNAPT, and APOL1. $n=6$ controls and 8 patients with HIVAN. $* * * P 0.001$ versus control. Original magnification: $\times 400$ (A, top row and middle row, B, and D); $\times 1000($ A, bottom row).
} 
coverslip were transfected with $\mathrm{EV}$ or an inhibitor of miR93a. After 48 hours, cells were labeled for PEC and transition markers. Cells were examined under a confocal microscope. Representative fluoromicrographs are shown in Figure 7D. miR193a inhibitor not only down-regulated the expression of PECs markers (PAX2 and claudin 1) but also up-regulated the expression of transition markers. These findings suggest that HIV-, IFN- $\gamma-$, and VDA-mediated APOL1 expression in PECs is mediated through downregulation of miR193a.

\section{AP0L1 Is Critical for the Functionality of APOL1-miR193a Axis in Human PECs}

Because the expression of APOL1 drives PECs to transition, it was tested whether lack of APOL1 could be indispensable for human PEC phenotype. PECs (undifferentiated) were transfected with scrambled or APOL1 siRNAs $(n=4)$. Protein blots were probed for PEC markers. Gels from three different lysate preparations are displayed (Figure 8A). Cumulative densitometric data are shown in a bar diagram (Figure 8B). APOL1 (mRNA) -silenced PECs displayed accentuated expression of PEC markers. These findings suggest that the lack of APOL1 optimizes PEC molecular phenotype.

To test whether APOL1 is necessary for the functionality of the APOL1-miR193a axis, PECs (undifferentiated) were transfected with scrambled or APOL1 siRNA. Control and experimental cells were treated with vehicle (control), VDR agonists, IFN- $\gamma$, or miR193a inhibitor or transduced with HIV $(n=4)$. Protein blots were probed for APOL1 and reprobed for transition markers. Representative gels are displayed in Figure 8, C and E. Cumulative densitometric data are shown in Figure 8, D and F. VDR agonists, IFN- $\gamma$, and miR193a inhibitor enhanced the expression of APOL1 and transition markers. However, the silencing of APOL1 down-regulated the expression of transition makers despite inhibition of miR193a. These findings suggest that lack of APOL1 expression ensures the PEC phenotype (which seems to be indispensable), whereas the presence and expression of APOL1 are needed in sustaining the transition of PECs (which seems to be dispensable but carries a trade-off).

\section{AP0L1 Is a Potential Target for miR193a}

The data suggest that APOL1 is a potential target for miR193a. To probe complementary APOL1, binding sites of miR193a were mapped by bioinformatics analysis (Figure 9A). HepG2 cells were transiently transfected with EV or miR193a plasmid and evaluated for APOL1 protein (Figure 9, B and C), APOL1 mRNA (Figure 9D), and miR193a expression (Figure 9E). In parallel sets of experiments, HEK cells were transfected with EV, pcMVmiR193a, pEZX-MT06 (control), or pEZx-MT06-APOL1 and evaluated for APOL1 luciferase signaling.
Overexpressing pCMV-miR193a displayed significantly decreased pEZX-MT06-APOL1 luciferase signal compared with EV (Figure 9F), suggesting a putative interaction between miR-193a and APOL1. Relative luciferase activity with different concentrations of pCMV-miR-193a is shown in Figure 9G. These findings suggest that there is a putative interaction between miR193a and APOL1.

\section{AP0L1 Induction in PECs in Vivo}

To determine whether HIV induces APOL1 in PECs in patients with HIVAN, co-labeling was performed for cytokeratin (PEC marker), synaptopodin (PD marker), and APOL1 in renal biopsy specimens of controls and patients with HIVAN. Representative fluoromicrographs are displayed in Figure 10. A glomerulus from a control patient did not show any expression of APOL1 in PECs.

PECs showed the expression of cytokeratin (green fluorescence). Podocytes displayed the expression of APOL1 in a glomerulus from a control patient. A glomerulus from a patient with HIVAN displayed robust APOL1 and cytokeratin expression by PECs (Figure 10A). PECs (cytokeratin labeled) co-labeled for APOL1 and synaptopodin in the glomerulus suggested a partial transition of PECs. Analysis of glomerular cells (CellProfiler) in controls and patients with HIVAN in the form of co-labeling for cytokeratin and APOL1 (Figure 10B) and triple labeling for cytokeratin, APOL1, and synaptopodin (Figure 10D) are shown as dot plots (Figure 10, C and E).

To confirm the role of APOL1 in PEC transition in vivo, renal cortical sections of HIV transgenic mice expressing APOL1 (G1) (Tg26: APOL1) and FVB/N (control) mice were co-labeled for APOL1 and synaptopodin $(n=4)$. Representative fluoromicrographs are shown in Figure 11A. Representative merged images using CellProfiler are shown in Figure 11B. Analysis of glomeruli (CellProfiler) in control and HIV transgenic mice expressing APOL1 is shown as dot plots (Figure 11C). Foci of PECs displayed expression of synaptopodin. These findings suggest that APOL1 carries the potential of stimulating PEC transition in vivo. Most podocytes showed co-labeling for synaptopodin and APOL1. In Tg26:APOL1 mice, foci of PECs also showed the expression for synaptopodin (Figure 11A).

A proposed schematic model of APOL1 dynamics in human PEC phenotype kinetics is shown in Figure 12. APOL1 and miR193a display a reciprocally linked feedback loop. Up-regulation of APOL1 and down-regulation of miR193a feed back to each other and initiate PECs transition; however, the lack of APOL1 with up-regulation of miR193a in a reciprocal manner governs PEC phenotype. Therefore, the APOL1 expression is dispensable unless or until there is a need for podocyte renewal. However, suppression of APOL1 expression in human PECs seems to be indispensable (to preserve their phenotype). 


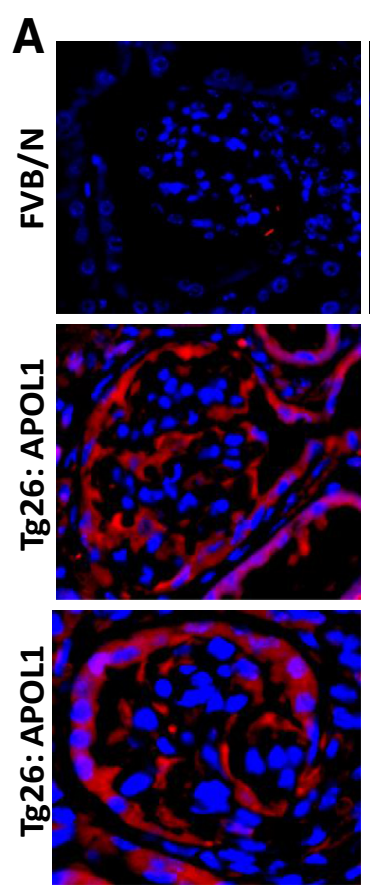

APOL1
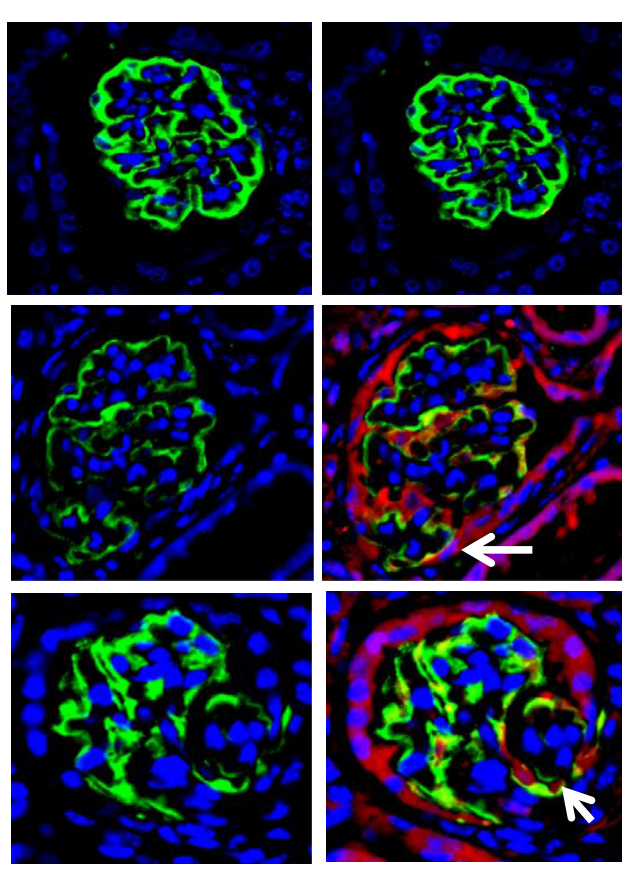

SYNAPT
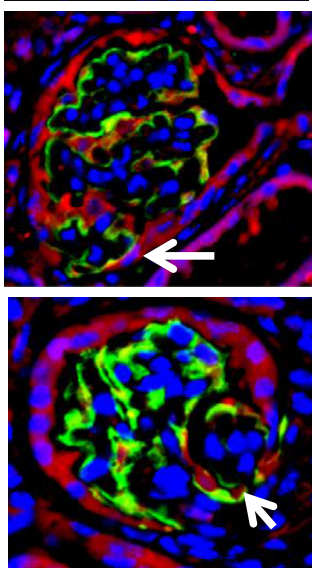

Merged

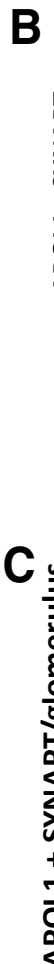

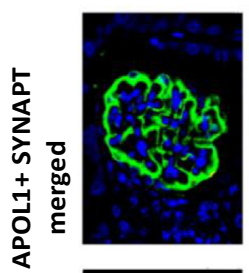

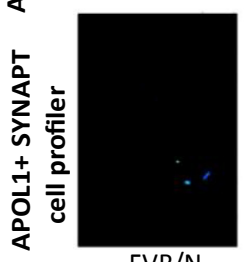

$\mathrm{FVB} / \mathrm{N}$
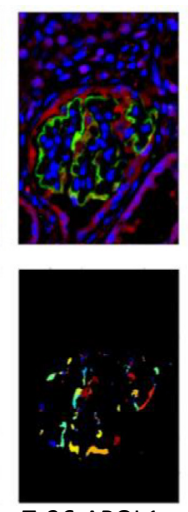

Tg26:APOL1

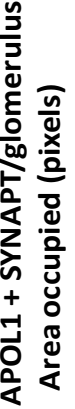

200,000
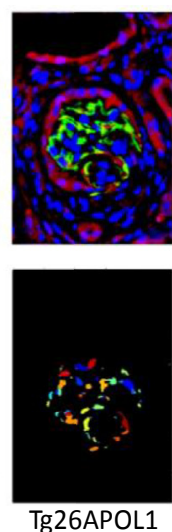

Tg26APOL1
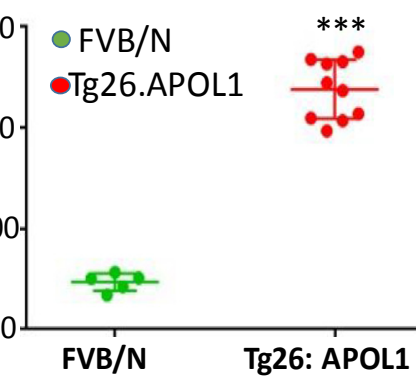

Figure 11 Podocyte expression of apolipoprotein (APO) L1 in Tg26:APOL1G1 transgenic mice. A: Renal cortical sections of control (FVB/N) and HIV transgenic mice expressing APOL1 (Tg26:APOL1) were co-labeled for APOL1 (red fluorescence) and synaptopodin (SYNAPT) (green fluorescence). Representative microfluorographs are shown. Foci of parietal epithelial cells (PECs) display expression of SYNAPT (arrows). B: Representative original images of a renal cortical section of control (FVB/N) and TG26:APOL1 mice showing expression of APOL1 (red fluorescence) and SYNAPT (green fluorescence) were captured using SlideBook software version 6.0. Images were then processed using CellProfiler pipeline to analyze co-expression of APOL1 and SYNAPT. The processed images show randomly denoted colors of the area occupied by pixels expressing SYNAPT and APOL1. C: Randomly selected glomeruli from FVB/N and TG26:AP0L1 mice were analyzed using CellProfiler. Data for the area occupied by pixels expressing SYNAPT and APOL1 were collected and analyzed using GraphPad Prism 7 software. A dot plot shows a comparison of the number of pixels co-expressing SYNAPT and AP0L1 between FVB/N and Tg26:AP0L1 mice. $n=4$. ${ }^{* * *} P<0.001$ versus FVB/N. Original magnification: $\times 400(\mathbf{A}) ; \times 200($ B $)$.

\section{Discussion}

In the present study, the role of the APOL1-miR193a axis in the PEC transition has been demonstrated in in vitro studies. APOL1 expression down-regulated but its silencing enhanced miR193a expression in PECs. APOL1-induced downregulation of miR193a was associated with the PECs transition, but APOL1 silencing enhanced the expression of miR193a and optimized PEC phenotype. Moreover, HEK cells (cells with undetectable APOL1 protein expression; negative control) transfected with APOL1 displayed the induction of the transition markers. Furthermore, podocyte APOL1 stimulators induced not only APOL1 expression in PECs but also the expression of the transition markers.

Mechanistic studies revealed that HIV, IFN- $\gamma$, and VDR agonists induced APOL1 expression in PECs through down-regulation of miR193a. As expected, inhibition of miR193a induced the expression of APOL1 in PECs. In HepG2 cells (non-kidney cells expressing APOL1), silencing of APOL1 enhanced miR193a expression, but overexpression of miR193a down-regulated the transcription of APOL1. A luciferase complementation-based interaction assay suggested a putative interaction between miR-193a and APOL1. Because silencing of APOL1 in PECs attenuated HIV-, VDR agonist-, miR193a inhibitorand IFN- $\gamma$-induced expression of transition markers, APOL1 is likely a critical functional constituent of the miR193-APOL1 axis in PECs. In brief, we found that APOL1 and miR193a are reciprocally linked regarding their expression in PECs, kidney cells with undetectable APOL1 protein expression (HEK cells, negative control), and non-kidney cells such as HepG2 cells, which express APOL1 (positive control). In addition, we found that PECs display APOL1 expression in patients with HIVAN.

Because the development of human machinery is presumably the last chain of evolution, the APOL1-miR193a axis must have evolved in a relatively short period of evolution, perhaps a couple of million years. We speculate that there may be an analog of APOL1 in other primates and mammals of which we are not aware. We stumbled on APOL1 because of its trypanolytic properties, and its mutants were found to be associated with a higher rate of chronic kidney diseases. ${ }^{1,6-12}$ Alternatively, it was an evolution for podocyte repair in human kidneys, which are highly active 


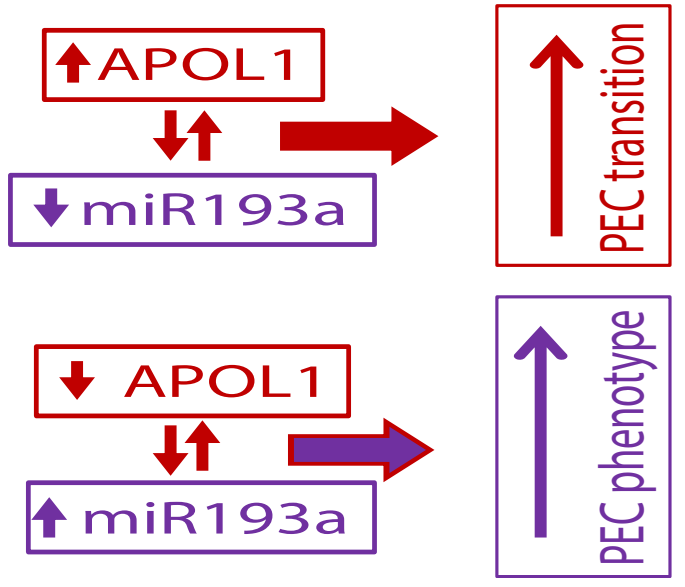

Figure 12 The proposed schematic hypothesis of apolipoprotein (AP0) L1 dynamics in human parietal epithelial cell (PEC) phenotype kinetics. APOL1 and miR193a display a reciprocally linked feedback loop. Upregulation of APOL1 and down-regulation of miR193a feedback each other and initiate PEC transition; conversely, down-regulation of APOL1 with up-regulation of miR193a in a reciprocal manner ensures the PEC phenotype.

and constantly overworking because of easy accessibility of food and water throughout the year for the last thousands of years. Nonetheless, currently, this is only conjecture, and more studies need to be conducted to probe this aspect.

Clinical reports suggest that APOL1 is dispensable except under pathogen attack. ${ }^{34}$ For example, rodents lack APOL1 but have normal podocyte function. In an isolated report, a human lacking APOL1 was found not to have any abnormality in his kidney function. ${ }^{34}$ In contrast, the present study suggests that APOL1 may well serve an important function in PECs. Under the normal physiologic state, suppression of APOL1 is crucial for sustaining PEC cellular phenotype. However, APOL1 emerges in PECs during their transition as a consequence of inhibited miR193a levels. Because most patients with focal segmental glomerulosclerosis have a higher level of miR193a in their glomeruli, ${ }^{35}$ PECs would neither express APOL1 nor undergo transition. On that account, APOL1 expression in PECs remained undetectable in pathologic states.

Structurally, PECs and podocytes are contiguous at the vascular pole. Notably, both the cells originate from the same mesenchymal cells during embryogenesis. ${ }^{20}$ PECs are the nearest bystander cells to podocytes, which are regulated by the same transcription factors, such as WT1 and miRNAs, such as miR193 $a^{20,35}$; moreover, both the cells face identical hostile environment-podocytes, and PECs are exposed to the same macromolecules/toxins passing through Bowman's space. Higher levels of miR193a (associated with adverse milieus) would be detrimental for the integrity of podocytes but would favor PEC survival. Because miR193a inversely regulates the expression of APOL1, loss of APOL1 seems to be the trade-off by PECs to preserve their integrity in a high-miR193a environment. Thus, it appears that the evolution prioritized the maintenance of the integrity of Bowman's capsule in high-miR193a milieus by turning off APOL1 expression in human PECs. On that account, the lack of APOL1 (to sustain high miR193a levels) could be considered an indispensable feature of human PECs. We propose that this trade-off prevents PEC participation in podocyte homeostasis during adverse milieus and contributes to the development of focal segmental glomerulosclerosis. Currently, this is only a speculation and requires further detailed studies.

Several investigators found APOL1 expression by kidney cells, including podocytes and tubular cells in certain physiologic and pathological states. ${ }^{1-3}$ These investigators did not find any expression of APOL1 by PECs in glomeruli of control subjects. Our observations in normal glomeruli are consistent with their findings. One can also justify the absence of APOL1 staining in PECs and attenuated staining in podocytes in renal biopsy specimens of focal segmental glomerulosclerosis in earlier reported studies. ${ }^{2}$ Patients with focal segmental glomerulosclerosis have a higher expression of miR193a in their glomeruli. ${ }^{35} \mathrm{~A}$ higher expression of miR193a would down-regulate the expression of $\mathrm{WT} 1^{4,33}$ and APOL $1,{ }^{4}$ resulting in enhanced expression of PAX2, a marker of PECs. ${ }^{4}$ Therefore, a higher level of miR193a would not only prevent PEC transition but would also induce dedifferentiation in podocytes, double jeopardy in podocyte homeostasis.

Notably, APOL1 expression in PECs was not reported in patients with HIVAN either. ${ }^{2}$ Most of the accumulated cells in Bowman's space in diseased glomeruli of patients with HIVAN are of PEC origin. ${ }^{36}$ Therefore, it is essential to label APOL1-expressing cells for cytokeratin (a PEC marker), to confirm their lineage. Because PECs are in transition mode in the HIV milieu, they would also display some podocyte markers; thus, co-labeling the cells (accumulated in Bowman's space in patients with HIVAN) for a podocyte marker will not help either. On that account, these cells were considered to be proliferating podocytes for a long time. ${ }^{37,38}$ Therefore, the only way to prove their PEC lineage is to label them for their cytokeratin expression. In our study, several APOL1 expressing cells in Bowman's space were positive for cytokeratin, indicating their PEC lineage. Therefore, although there is no discrepancy between our findings and observations by other investigators, ${ }^{2}$ there are technical issues that do not allow a direct comparative analysis between the current and the cited study.

PECs have been demonstrated to participate in podocyte homeostasis in mice, which do not have APOL1 in their genome $^{6-8}$; of note, the numbers of PECs capable of transiting to podocytes are limited, and their transition is limited to the juvenile period. ${ }^{7,8}$ It is plausible that most PECs in adult mice are not capable of participating in podocyte homeostasis as a consequence of the lack of APOL1. It would be worth studying this aspect in APOL1 transgenic mice in future studies.

In summary, both APOL1 and miR193a are reciprocally linked with a feedback loop in human PECs. Up-regulation 
of miR193a sustains the PEC phenotype through down-regulation of APOL1, whereas down-regulation of miR193a initiates PEC transition through up-regulation of APOL1.

\section{Acknowledgments}

We thank Prof. Sanjeev Gupta (Albert Einstein College Medicine) for kindly providing the HepG2 cells and Prof. Paul Klotman (University Texas) for kindly providing the Tg26 mice.

\section{Supplemental Data}

Supplemental material for this article can be found at https://doi.org/10.1016/j.ajpath.2018.07.025.

\section{References}

1. Vanhamme L, Paturiaux-Hanocq F, Poelvoorde P, Nolan DP, Lins L, Van Den Abbeele J, Pays A, Tebabi P, Van Xong H, Jacquet A, Moguilevsky N, Dieu M, Kane JP, De Baetselier P, Brasseur R, Pays E: Apolipoprotein L-I is the trypanosome lytic factor of human serum. Nature 2003, 422:83-87

2. Madhavan SM, O'Toole JF, Konieczkowski M, Ganesan S, Bruggeman LA, Sedor JR: APOL1 localization in normal kidney and nondiabetic kidney disease. J Am Soc Nephrol 2011, 22:2119-2128

3. Ma L, Shelness GS, Snipes JA, Murea M, Antinozzi PA, Cheng D, Saleem MA, Satchell SC, Banas B, Mathieson PW, Kretzler M, Hemal AK, Rudel LL, Petrovic S, Weckerle A, Pollak MR, Ross MD, Parks JS, Freedman BI: Localization of APOL1 protein and mRNA in the human kidney: nondiseased tissue, primary cells, and immortalized cell lines. J Am Soc Nephrol 2015, 26:339-348

4. Mishra A, Ayasolla K, Kuma V, LAN X, Vashistha H, Aslam R, Hussain A, Chowdhary S, Shoshtari SM, Paliwal N, Popik W, Saleem MA, Malhotra A, Meggs LG, Skorecki K, Singhal PC: Modulation of apolipoprotein L1- microRNA-193a axis prevents podocyte dedifferentiation in high-glucose milieu. Am J Physiol Renal Physiol 2018, 314:F832-F843

5. Lee H, Roshanravan H, Wang Y, Okamoto K, Ryu J, Shrivastav S, Qu P, Kopp JB: APOL1 renal risk variants induce aberrant THP-1 monocyte differentiation and increase eicosanoid production via enhanced expression of cyclooxygenase-2. Am J Physiol Renal Physiol 2018, 315:F140-F150

6. Kopp JB, Nelson GW, Sampath K, Johnson RC, Genovese G, An P, Friedman D, Briggs W, Dart R, Korbet S, Mokrzycki MH, Kimmel PL, Limou S, Ahuja TS, Berns JS, Fryc J, Simon EE, Smith MC, Trachtman H, Michel DM, Schelling JR, Vlahov D, Pollak M, Winkler CA: APOL1 genetic variants in focal segmental glomerulosclerosis and HIV-associated nephropathy. J Am Soc Nephrol 2011, 22:2129-2137

7. Friedman DJ, Kozlitina J, Genovese G, Jog P, Pollak MR: Populationbased risk assessment of APOL1 on renal disease. J Am Soc Nephrol 2011, 22:2098-2105

8. Freedman BI, Kopp JB, Langefeld CD, Genovese G, Friedman DJ, Nelson GW, Winkler CA, Bowden DW, Pollak MR: The apolipoprotein L1 (APOL1) gene and nondiabetic nephropathy in African Americans. J Am Soc Nephrol 2010, 21:1422-1426

9. Larsen CP, Freedman BI: Apolipoprotein L1-associated nephropathy and the future of renal diagnostics. J Am Soc Nephrol 2015, 26: $1232-1235$
10. Grams ME, Rebholz CM, Chen Y, Rawlings AM, Estrella MM, Selvin E, Appel LJ, Tin A, Coresh J: Race, APOL1 risk, and eGFR decline in the general population. J Am Soc Nephrol 2016, 27: $2842-2850$

11. Dummer PD, Limou S, Rosenberg AZ, Heymann J, Nelson G, Winkler CA, Kopp JB: APOL1 kidney disease risk variants - an evolving landscape. Semin Nephrol 2015, 35:222-236

12. Limou S, Nelson GW, Kopp JB, Winkler CA: APOL1 kidney risk alleles: population genetics and disease associations. Adv Chronic Kidney Dis 2014, 21:426-433

13. Shankland SJ, Smeets B, Pippin JW, Moeller MJ: The emergence of the glomerular parietal epithelial cell. Nat Rev Nephrol 2014, 10: $158-173$

14. Appel D, Kershaw DB, Smeets B, Yuan G, Fuss A, Frye B, Elger M, Kriz W, Floege J, Moeller MJ: Recruitment of podocytes from glomerular parietal epithelial cells. J Am Soc Nephrol 2009, 20: 333-343

15. Berger K, Schulte K, Boor P, Kuppe C, van Kuppevelt TH, Floege J, Smeets B, Moeller MJ: The regenerative potential of parietal epithelial cells in adult mice. J Am Soc Nephrol 2014, 25:693-705

16. Lazzeri E, Romagnani P: Podocyte biology: differentiation of parietal epithelial cells into podocytes. Nat Rev Nephrol 2015, 11:7-8

17. Eng DG, Sunseri MW, Kaverina NV, Roeder SS, Pippin JW, Shankland SJ: Glomerular parietal epithelial cells contribute to adult podocyte regeneration in experimental focal segmental glomerulosclerosis. Kidney Int 2015, 88:999-1012

18. Shankland SJ, Freedman BS, Pippin JW: Can podocytes be regenerated in adults? Curr Opin Nephrol Hypertens 2017, 26:154-164

19. Roeder SS, Stefanska A, Eng DG, Kaverina N, Sunseri MW, McNicholas BA, Rabinovitch P, Engel FB, Daniel C, Amann K, Lichtnekert J, Pippin JW, Shankland SJ: Changes in glomerular parietal epithelial cells in mouse kidneys with advanced age. Am J Physiol Renal Physiol 2015, 309:F164-F178

20. Kietzmann L, Guhr SS, Meyer TN, Ni L, Sachs M, Panzer U, Stahl RA, Saleem MA Kerjaschki D, Gebeshuber CA, MeyerSchwesinger C: MicroRNA-193a regulates the transdifferentiation of human parietal epithelial cells toward a podocyte phenotype. J Am Soc Nephrol 2015, 26:1389-1401

21. Miesen L, Steenbergen E, Smeets B: Parietal cells: new perspectives in glomerular disease. Cell Tissue Res 2017, 369:237-244

22. Husain M, Meggs LG, Vashistha H, Simoes S, Griffiths KO, Kumar D, Mikulak J, Mathieson PW, Saleem MA, Del Valle L, PinaOviedo S, Wang JY, Seshan SV, Malhotra A, Reiss K, Singhal PC: Inhibition of p66ShcA longevity gene rescues podocytes from HIV1-induced oxidative stress and apoptosis. J Biol Chem 2009, 284: $16648-16658$

23. Chandel N, Sharma B, Husain M, Salhan D, Singh T, Rai P, Mathieson PW, Saleem MA, Malhotra A, Singhal PC: HIV compromises integrity of podocyte actin cytoskeleton through down regulation of vitamin D receptor. Am J Physiol Renal Physiol 2013, 304: F1347-F1357

24. Kumar D, Konkimalla S, Yadav A, Sataranatarajan K, Kasinath BS, Chander PN, Singhal PC: HIV-associated nephropathy: role of mammalian target of rapamycin pathway. Am J Pathol 2010, 177: 813-821

25. Kopp JB, Ray PE, Adler SH, Bruggeman LA, Mangurian CV, Owens JW, Eckhaus MA, Bryant JL, Klotman PE: Nephropathy in HIV-transgenic mice. Contrib Nephrol 1994, 107:194-204

26. Klotman PE, Rappaport J, Ray P, Kopp JB, Franks R, Bruggeman LA, Notkins AL: Transgenic models of HIV-1. AIDS 1995, 9:313-324

27. Carpenter AE, Jones TR, Lamprecht MR, Clarke C, Kang IH, Friman O, Guertin DA, Chang JH, Lindquist RA, Moffat J, Golland P, Sabatini DM: CellProfiler: image analysis software for identifying and quantifying cell phenotypes. Genome Biol 2006, 7:R100

28. Michael RL, David MS, Anne EC: CellProfiler ${ }^{\mathrm{TM}}$ : free, versatile software for automated biological image analysis. Biotechniques 2007, 42:71-75 
29. Haque S, Patil G, Mishra A, Lan X, Popik W, Malhotra A, Skorecki K, Singhal PC: Effect of APOL1 disease risk variants on APOL1 gene product. Biosci Rep 2017, 37. BSR20160531

30. Mikulak J, Oriolo F, Portale F, Tentorio P, Lan X, Saleem MA, Skorecki K, Singhal PC, Mavilio D: Impact of APOL1 polymorphism and IL-1 $\beta$ priming in the entry and persistence of HIV-1 in human podocytes. Retrovirology 2016, 13:63

31. Lan X, Jhaveri A, Cheng K, Wen H, Saleem MA, Mathieson PW, Mikulak J, Aviram S, Malhotra A, Skorecki K, Singhal PC: APOL1 risk variants enhance podocyte necrosis through compromising lysosomal membrane permeability. Am J Physiol Renal Physiol 2014, 307: F326-F336

32. Lan X, Wen H, Lederman R, Malhotra A, Mikulak J, Popik W, Skorecki K, Singhal PC: Protein domains of APOL1 and its risk variants. Exp Mol Pathol 2015, 99:139-144

33. Tzur S, Rosset S, Shemer R, Yudkovsky G, Selig S, Tarekegn A, Bekele E, Bradman N, Wasser WG, Behar DM, Skorecki K: Missense mutations in the APOL1 gene are highly associated with end-stage kidney disease risk previously attributed to the MYH9 gene. Hum Genet 2010, 128:345-350
34. Johnstone DB, Shegokar V, Nihalani D, Rathore YS, Mallik L, Ashish, Zare V, Ikizler HO, Powar R, Holzman LB: APOL1 null alleles from a rural village in India do not correlate with glomerulosclerosis. PLoS One 2012, 7:e51546

35. Gebeshuber CA, Kornauth C, Dong L, Sierig R, Seibler J, Reiss M, Tauber S, Bilban M, Wang S, Kain R, Böhmig GA, Moeller MJ, Gröne HJ, Englert C, Martinez J, Kerjaschki D: Focal segmental glomerulosclerosis is induced by microRNA-193a and its downregulation of WT1. Nat Med 2013, 19:481-487

36. Dijkman HB, Weening JJ, Smeets B, Verrijp KC, van Kuppevelt TH, Assmann KK, Steenbergen EJ, Wetzels JF: Proliferating cells in HIV and pamidronate- associated collapsing focal segmental glomerulosclerosis are parietal epithelial cells. Kidney Int 2006, 70:338-344

37. Barisoni L, Mokrzycki M, Sablay L, Nagata M, Yamase H, Mundel P: Podocyte cell cycle regulation and proliferation in collapsing glomerulopathies. Kidney Int 2000, 58:137-143

38. Yang Y, Gubler MC, Beaufils H: Dysregulation of podocyte phenotype in idiopathic collapsing glomerulopathy and HIV-associated nephropathy. Nephron 2002, 91:416-423 